\title{
Reconstitution of $\beta$-adrenergic regulation of Cav1.2: Rad-dependent and Rad-independent protein kinase A mechanisms.
}

Short title: Reconstitution of $\beta$-adrenergic regulation of Cav1.2

Moshe Katz ${ }^{1}$, Suraj Subramaniam ${ }^{2}$, Orna Chomsky-Hecht ${ }^{2}$, Vladimir Tsemakhovich ${ }^{1}$, Anouar Belkacemi ${ }^{4}$, Veit Flockerzi ${ }^{4}$, Enno Klussmann ${ }^{5}$, Joel A. Hirsch ${ }^{2,3}$, Sharon Weiss ${ }^{1}$, Nathan Dascal ${ }^{1,3}$

${ }^{1}$ Sackler School of Medicine, ${ }^{2}$ Faculty of Life Sciences, ${ }^{3}$ Sagol School of Neuroscience, Tel Aviv University, Israel; ${ }^{4}$ Experimentelle und Klinische Pharmakologie und Toxikologie, Universitat des Saarlandes, 66421, Homburg, Germany; ${ }^{5}$ Max Delbrück Center for Molecular Medicine (MDC) in the Helmholtz Association, Berlin, Germany.

Correspondence to: Nathan Dascal, School of Medicine, Tel Aviv University, Ramat Aviv, Tel Aviv 6997801, Israel.

tel. (972) 3 6405743; fax. (972) 3 6409113; e-mail: dascaln@tauex.tau.ac.il 


\section{Abstract \\ Introduction}

Cardiac L-type voltage-gated Cav1.2 channels are crucial in physiological regulation of cardiac excitation-contraction coupling. Adrenergic modulation of Cav1.2 starts with activation of $\beta$ adrenergic receptors (AR) and culminates in protein kinase $A(P K A)$ - induced increase of calcium influx through Cav1.2 channels. To date, this cascade has never been fully reconstituted in heterologous systems; even partial reconstitution proved challenging and controversial. A recent study identified Rad, a calcium channel inhibitory protein, as an essential component of the adrenergic signaling cascade. We corroborated this finding, further characterized, and fully reconstituted, the complete $\beta$-AR Cav1.2 modulation cascade in a heterologous expression system.

\section{Objective}

Our primary goal was to heterologously reconstitute the complete $\beta$-adrenergic cascade, and to investigate the role of Rad and additional molecular determinants in adrenergic regulation of cardiac Cav1.2.

Methods and Results: We utilized the Xenopus oocyte heterologous expression system. We expressed Cav1.2 channel subunits, without or with Rad and $\beta 1-A R$ or $\beta 2-A R$. To activate PKA, we injected cyclic AMP (cAMP) into the oocytes, or extracellularly applied isoproterenol (Iso) to stimulate $\beta$-AR. Whole-cell $\mathrm{Ba}^{2+}$ currents served as readout. We find and distinguish between two distinct pathways of PKA modulation of Cav1.2: Rad-dependent ( $80 \%$ of total) and Radindependent. We separate the two mechanisms by showing distinct requirements for the cytosolic N- and distal C- termini of $\alpha_{1 c}$ and for the Cav $\beta$ subunit. Finally, for the first time, we reconstitute the complete pathway using agonist activation of either $\beta 1-A R$ or $\beta 2-A R$. The reconstituted system reproduces the known features of $\beta$-AR regulation in cardiomyocytes, such as a >2-fold increase in Cav1.2 current, a hyperpolarizing shift in activation curve, and a high constitutive activity of $\beta 2-A R$.

\section{Conclusions}

The adrenergic modulation of Cav1.2 is composed of two distinct pathways, Rad-independent and Rad-dependent. The latter contributes most of the $\beta$-AR-induced enhancement of Cav1.2 
activity, crucially depends on $C_{v} \beta$ subunit, and is differently regulated by $\beta 1-A R$ and $\beta 2-A R$. The reconstitution of the full $\beta$-AR cascade provides the means to address central unresolved issues related to roles of auxiliary proteins in the cascade, Cav1.2 isoforms, and will help to develop therapies for catecholamine-induced cardiac pathologies.

\section{Introduction}

Cardiac excitation-contraction coupling crucially depends on the L-type voltage dependent $\mathrm{Ca}^{2+}$ channel, Cav1.2. Influx of extracellular $\mathrm{Ca}^{2+}$ via Cav1.2 triggers $\mathrm{Ca}^{2+}$ release from the sarcoplasmic reticulum, via the $\mathrm{Ca}^{2+}$ release channel (ryanodine receptor 2$)^{1}$. Activation of the sympathetic nervous system increases heart rate, relaxation rate and contraction force. The latter is due largely to increased $\mathrm{Ca}^{2+}$ influx via Cav1.2 ${ }^{2}$. Pathological prolonged sympathetic activation progressively impairs cardiac function, causing heart failure, partly due to misregulation of $\mathrm{Ca}_{\mathrm{v}} 1.2^{3}$.

Cardiac Cav1.2 is a heterotrimer comprising the pore-forming subunit $\alpha_{1 \mathrm{c}}(\sim 240 \mathrm{kDa})$, the intracellular $C a_{\vee} \beta_{2}(\sim 68 \mathrm{kDa})$ and the extracellularly located $\alpha 2 \delta(\sim 170 \mathrm{kDa})^{4,5}$. The $\mathrm{N}$ and $\mathrm{C}$ termini (NT, CT respectively) of $\alpha_{1 \mathrm{C}}$ are cytosolic and vary among Cav1.2 isoforms (Fig. 1A). It is believed that most of the cardiac $\alpha_{1 c}$ protein is post-translationally cleaved at the CT, around amino acid (a.a.) 1800 , to produce the truncated $\sim 210 \mathrm{kDa} \alpha_{1 \mathrm{c}}$ protein and the $\sim 35 \mathrm{kDa}$ cleaved distal CT (dCT); however, the full-length protein is also present ${ }^{6-9}$.

The sympathetic nervous system activates cardiac $\beta$-adrenergic receptors ( $\beta-A R)$, primarily $\beta 1-A R$ (which mediates most of the $\beta A R$-enhancement of contraction and Cav1.2 activity) and $\beta 2-A R^{10}$. $\beta 1-A R$ is globally distributed in cardiomyocytes, couples to $G_{s}$ and elevates CAMP levels within the whole cardiomyocyte ${ }^{10}$. In contrast, $\beta 2-A R$ shows spatially restricted localization to the T-tubules (which changes in heart failure) ${ }^{11}$ and couples to $\mathrm{G}_{\mathrm{s}}$ and $\mathrm{G}_{\mathrm{i} / \mathrm{o}}{ }^{12}$, producing localized cAMP increases ${ }^{3}$.

The cascade of adrenergic modulation of Cav1.2 comprises agonist binding to $\beta$-ARs, activation of $\mathrm{G}_{\mathrm{s}}$, adenylyl cyclase, elevated intracellular cAMP levels, and activation of protein kinase $A$ (PKA). The PKA holoenzyme consists of two regulatory subunits (PKA-RS) bound to two catalytic subunits (PKA-CS). CAMP binds to PKA-RS and causes dissociation of PKA-CS and PKA- 
CS. The latter enhances Cav1.2 activity. However, the final step, how PKA-CS enhances Cav1.2 activity, remained enigmatic. A long standing paradigm was a direct phosphorylation by PKA-CS of $\alpha_{1 c}$ and/or Cav $\beta$ subunits ${ }^{2}$. However, numerous studies critically challenged this theory. In particular, mutated Cav1.2 channels in genetically engineered mice lacking putative PKA phosphorylation sites on $\alpha_{1 c}$ and/or $\beta_{2 b}$, were still upregulated by PKA ${ }^{7,13-16}$ (reviewed in ${ }^{4,17}$ ).

One significant obstacle in deciphering the mechanism of PKA regulation of Cav1.2 was a recurrent failure to reconstitute the regulation in heterologous systems, which proved challenging and controversial ${ }^{18}$. Previous studies in heterologous cellular models, including Xenopus oocytes, demonstrated that cAMP failed to up-regulate Cav1.2 containing the fulllength $\alpha_{1 C}, \mathrm{Cav} 1.2-\alpha_{1 C}{ }^{19-21}$. However, robust $\beta-A R$ - induced upregulation of $\mathrm{Ca}^{2+}$ currents was observed in oocytes injected with total heart RNA ${ }^{22}$, suggesting the necessity of an auxiliary protein, the "missing link"19, 20 . Interestingly, partial regulation was observed with dCTtruncated $\alpha_{1 c}{ }^{23,24}$, which is considered the predominant form of $\alpha_{1 c}$ in the heart ${ }^{2}$. We have previously reported a modest (30-40\%) upregulation of Cav1.2, containing a dCT-truncated $\alpha_{1 C}$, by intracellular injection of CAMP or PKA-CS in Xenopus oocytes ${ }^{24}$. Additionally, this regulation required the presence of the initial segment of the long-NT of $\alpha_{1 C}$, but did not involve Cav $\beta$ subunit. We proposed that this mechanism may account for part of the adrenergic regulation of Cav1.2 in the heart ${ }^{24}$. Normally adrenergic stimulation in cardiomyocytes increases the Ca ${ }^{2+}$ current two to three fold, thus a major part of the regulation has remained unexplained.

Recently, Liu et al. identified Rad as the "missing link" in PKA regulation of Cav1.2 ${ }^{15}$. Rad is a member of the Ras-related GTPase subfamily (RGK) that inhibits high voltage activated calcium channels Cav1 and Cav $2^{25}$. Rad tonically inhibits Cav1.2, largely via an interaction with $C_{v} \beta^{26,27}$. Ablation of Rad in murine heart was shown to increase basal Cav1.2 activity but suppressed $\beta$-AR regulation ${ }^{28}$. Liu et al. demonstrated that PKA phosphorylation of Rad relieves this tonic inhibition to increase Cav1.2 activity $^{15}$. They reconstituted a major part of the Cav1.2 regulation cascade, initiated by forskolin-activated adenylyl cyclase and ultimately attaining a $\sim 2$-fold increase in $\mathrm{Ca}^{2+}$ current, in mammalian cells expressing $\alpha_{1 C}, \alpha 2 \delta, \beta_{2 b}$ and $\operatorname{Rad}^{15}$. The relation between this Rad-dependent regulation and the regulation reported in our previous 
study ${ }^{24}$ is not clear. Furthermore, the complete pathway of adrenergic modulation of Cav1.2, starting with $\beta$-AR activation, has not yet been heterologously reconstituted.

Here we utilized the Xenopus oocyte heterologous expression system and, for the first time, reconstituted the entire pathway. We find and distinguish between two distinct pathways of PKA modulation of Cav1.2: a Rad-dependent and a Rad-independent pathway. We characterize the involvement of the $\mathrm{N}$ - and $\mathrm{C}$ - termini of $\alpha_{1 \mathrm{c}}$ and of the $\beta_{2 b}$ subunit in this crucial physiological regulation, and compare and contrast the Cav1.2 regulation by $\beta 1-A R$ or $\beta 2-A R$. Our findings reveal novel aspects of the roles of $\alpha_{1 c}$ (particularly the $\mathrm{N}$ - and $\mathrm{C}$-termini), $\beta_{2 b}$ and Rad in the adrenergic modulation of cardiac Cav1.2 channels. Reproducing the complete $\beta$-AR cascade in a heterologous expression system will promote the identification and characterization of intracellular proteins that regulate the cascade, eventually assisting efforts to develop therapies to treat heart failure and other catecholamine-induced cardiac pathologies.

\section{Methods}

\section{Experimental animals and ethical approval}

Oocytes were harvested from adult female Xenopus laevis frogs, as described ${ }^{24}$. Ethical approval was granted by Tel Aviv University Institutional Animal Care and Use Committee (permits 01-16-104 and 01-20-083).

\section{DNA constructs and RNA}

In this work we expressed Cav1.2 in Xenopus oocytes, usually in full subunit composition $\alpha_{1 c}+\alpha 2 \delta+\beta_{2 b}$, by injection of equal amounts, by weight, of RNAs of each subunit (except experiments of Fig. 2 that addressed the role of the Cav $\beta$ subunit). We used the long-NT isoform of rabbit $\alpha_{1 c}$ (except Fig. S1 where mouse $\alpha_{1 c}$ was used) and various mutants, as detailed in the figures. RNAs of $\beta 1-A R, \beta 2-A R$, human Rad, or the $\alpha_{1 C} d C T$ (as a separate protein) were expressed according to the design of the experiment. The Cav $\beta$ subunit used here was rabbit $\beta_{2 b}$ (originally termed $\beta_{2 a}{ }^{29}$ ). We tested the full-length $\beta_{2 b}$ and $\beta_{2 b}$-core, composed of a.a. 26-422 of Cav $\beta$ with deletion of the linker sequence (a.a. 138-202) as previously described ${ }^{30}$. The $\beta_{2 b^{-}}$ core $_{3 \mathrm{DA}}$ mutant ${ }^{31}$ was prepared based on the $\beta_{2 b}$-core construct with three point mutations 
(D244A/D320A/D322A) (see Fig. 2A). RNAs of all constructs were prepared and injected into oocytes as described ${ }^{24}$.

\section{Electrophysiology}

Recordings were performed on defolliculated oocytes 3-4 days post RNA injection. Whole-cell $\mathrm{Ba}^{2+}$ currents via Cav1.2 ( $\left.\mathrm{I}_{\mathrm{Ba}}\right)$ were recorded using two-electrode voltage clamp, routinely in a $40 \mathrm{mM} \mathrm{Ba}^{2+}$ solution (in $\mathrm{mM}: 40 \mathrm{Ba}(\mathrm{OH})_{2}, 50 \mathrm{NaOH}, 2 \mathrm{KOH}$, and 5 Hepes, titrated to $\mathrm{pH} 7.5$ with methanesulfonic acid). The membrane potential was $-80 \mathrm{mV}$ and $\mathrm{I}_{\mathrm{Ba}}$ was elicited by steps to $+20 \mathrm{mV}$ for $20 \mathrm{~ms}$ every $10 \mathrm{~s}$. To obtain the current-voltage (I-V) relationship, currents were elicited by $20 \mathrm{~ms}$ pulses starting at $-70 \mathrm{mV}$ up to $+80 \mathrm{mV}$ in $10 \mathrm{mV}$ increments every $10 \mathrm{~s}$. Currents measured in the presence of $200 \mu \mathrm{M} \mathrm{Cd}^{2+}$ were subtracted from total $\mathrm{I}_{\mathrm{Ba}}$ (Fig. 5A-C) to yield the net $\mathrm{I}_{\mathrm{Ba}}$. I-V curves were fitted using the Boltzmann equation ${ }^{32}$. The parameters obtained for $G_{\max }$ and $V_{\text {rev }}$ were then used to calculate fractional conductance at each $V_{m}$ and to construct the conductance-voltage $(G-V)$ curve. CFTR currents were measured at $-80 \mathrm{mV}$ in ND96 solution (in mM: $96 \mathrm{NaCl}, 2 \mathrm{KCl}, 1 \mathrm{MgCl}_{2}, 1 \mathrm{CaCl}_{2}, 5$ Hepes, pH 7.6).

CAMP, PKA or Iso were applied after verifying that $I_{\mathrm{Ba}}$ is stable for at least $2 \mathrm{~min}$. CAMP or PKA-CS were introduced into oocytes by pressure microinjection to final concentrations within the oocyte of $\sim 100 \mu \mathrm{M}$ (CAMP) or $\sim 25 \mathrm{ng} /$ oocyte (PKA-CS). Isoprenaline hydrochloride (isoproterenol, Iso) was perfused into the experimental chamber.

\section{Statistical analysis}

Results are presented as median and interquartile range (IQR) [Q1-Q3], or as mean \pm standard error for normally distributed continuous variables. For comparisons between groups, we used Student's t-test or Mann-Whitney test, as appropriate. The fold change in current caused by a treatment in a single oocyte was calculated as ( $I_{\mathrm{Ba}}$ after treatment $) /\left(I_{\mathrm{Ba}}\right.$ before treatment). The $\mathrm{I}_{\mathrm{Ba}}$ amplitudes before and after treatment in a single cell were compared using paired t-test or Wilcoxon test, as appropriate. For multiple group comparisons, we performed a One-Way ANOVA or Kruskal Wallis ANOVA on ranks, as appropriate. A Bonferroni post hoc test was performed for normally distributed data (Shapiro-Wilk test) and Dunnett's post hoc test otherwise. Statistical analysis was performed with SigmaPlot 13 (Systat Software Inc., San Jose, CA, USA). 


\section{Results}

\section{Rad plays a significant role in PKA regulation of $\mathrm{Ca}_{v} 1.2$}

PKA regulation of heterologously expressed Cav1.2 was previously observed only with dCT-truncated, but not full-length $\alpha_{1 C}{ }^{23,24}$. In Xenopus oocytes, $\mathrm{Ba}^{2+}$ currents (I $\mathrm{I}_{\mathrm{Ba}}$ ) via Cav1.2 containing dCT-truncated $\alpha_{1 C}\left(\operatorname{Cav} 1.2-\alpha_{1} \Delta 1821\right)$ were increased by $20-40 \%$ following intracellular injection of CAMP or PKA-CS ${ }^{24}$. Therefore, we started the study of Rad's role in regulation of $\mathrm{Ca}_{\vee} 1.2$ using $\alpha_{1 \mathrm{c}} \Delta 1821$.

We expressed Cav1.2 in full subunit composition, $\alpha_{1 c} \Delta 1821, \beta_{2 b}$ and $\alpha 2 \delta$, unless indicated otherwise, at a 1:1:1 RNA ratio (by weight), and tested the effect of increasing Rad concentrations by injecting varying amounts of Rad RNA. I $\mathrm{Ba}_{\mathrm{Ba}}$ was measured 3-5 days after RNA injection, by voltage steps from -80 to $+20 \mathrm{mV}$ (see Fig. 1C, inserts, for examples of $\mathrm{I}_{\mathrm{Ba}}$ recordings). As expected ${ }^{26,31}$, basal $\mathrm{I}_{\mathrm{Ba}}$ was reduced by expression of Rad, showing an inverse correlation with Rad RNA dose (Fig. 1B). Without Rad coexpression, $\mathrm{I}_{\mathrm{Ba}}$ was increased by $19 \% \pm 3$ $(n=32, p=0.002)$ following cAMP injection (Fig. 1C-E). Coexpression of Rad dramatically augmented the effect of CAMP (Fig. 1C, E). This effect of Rad became statistically significant at Rad: $\beta_{2 b}$ RNA ratios above 1:10, with a maximum increase of $127 \pm 10 \%$ at 1:2 ratio (Fig. 1F).

\section{The role of $\mathrm{Ca}_{v} \mathrm{~B}$ in Rad dependent regulation}

Rad inhibits Cav1.2 through $\operatorname{Cav} \beta$-dependent and $\operatorname{Cav} \beta$-independent mechanisms ${ }^{26,31}$. To test the role of Cav $\beta$ in PKA regulation of Cav1.2, we compared the change in $\mathrm{I}_{\mathrm{Ba}}$ following injection of cAMP into oocytes co-expressing $\alpha_{1 c} \Delta 1821, \alpha 2 \delta$, and $\beta_{2 b}$-wt (wild-type), $\beta_{2 b}$-core ${ }^{30}$, $\beta_{2 b}$-core $3 D A$ or no $\beta_{2 b}$, with or without Rad (Fig. 2A). $\beta_{2 b}$-core 3 BA was designed on the basis of $\beta_{2 b^{-}}$ core and contained the triple mutation D244A/D320A/D322A which abolishes CavB-Rad association $^{31,35}$.

In oocytes that did not express Rad, CAMP induced the typical, mild but statistically significant, 20-30\% increase in I $\mathrm{Ba}$ (Fig. 2B-D). This regulation is termed hereafter "Radindependent". The Rad-independent CAMP effect was similar in oocytes that did not express any Cav $\beta$ or expressed $\beta_{2 b}, \beta_{2 b}$-core, or the $\beta_{2 b}$-core 3 DA mutant (Fig. 2B-D). Co-expression of Rad 
resulted in a much larger, $\sim 2.5$-fold increase in peak currents, but only when $\beta_{2 b}$-wt or $\beta_{2 b}$-core were present (Fig. 2B, 2D). We refer to this regulation as "Rad-dependent".

Importantly, in Rad-expressing oocytes that did not express $\operatorname{Cav} \beta$, or expressed the $\beta_{2 b^{-}}$ core $_{3 \mathrm{DA}}$ mutant, cAMP caused only a mild increase in $\mathrm{I}_{\mathrm{Ba}}$ that resembled the Rad-independent cAMP effect (Fig. 2B-D). Notably, basal $\mathrm{I}_{\mathrm{Ba}}$ was significantly increased by coexpression of $\beta_{2 b^{-}}$ core $_{3 \mathrm{DA}}$ (e.g. Fig. $2 \mathrm{C}$ ) indicating robust protein expression and channel regulation. Evidently, abrogation of $\beta_{2 b}$-Rad interaction eliminates the Rad-dependent PKA regulation of Cav1.2. Thus, Rad-dependent PKA regulation of Cav1.2 is Cav $\beta$-dependent. $\beta_{2 b}$-core is sufficient to mediate the regulation. In contrast, the Rad-independent mechanism does not require Cav $\beta$ (as shown before $^{24}$ ) and its contribution to CAMP-induced current increase is much smaller than that of the Rad-dependent one.

\section{The N-terminus of $\alpha_{1 c}$ is important for Rad-independent but not Rad-dependent regulation}

We then set out to characterize the determinants of Rad-independent regulation. The predominant cardiac isoform is the long-NT $\alpha_{1 c}$, where the first 46 a.a., out of $\sim 154$, are encoded by the alternative exon $1 a^{34}$. The initial segment (first 20 a.a.) of this $\alpha_{1 c}$ isoform acts as an inhibitory module, tonically reducing the activity of Cav1.2 by reducing the channel's open probability $^{32}$. Within the NT initial segment, a.a. 6-20 show partial homology with the first 16 a.a. of the short NT isoform (these 16 a.a. are encoded by the alternative exon 1), found in smooth muscle and brain ${ }^{4}$ (Fig. S1A). Specifically, a.a. $T_{10}, Y_{13}$ and $P_{15}$ (TYP motif) are highly conserved and crucial for the inhibitory function of the NT module ${ }^{32}$. Importantly, the Radindependent PKA regulation of $\mathrm{Ca}_{v} 1.2-\alpha_{1 \mathrm{c}} \Delta 1821$ is greatly reduced or abolished by the removal of the first 5 or 20 a.a. of the long $\mathrm{NT}^{24}$. We have further addressed the role of the NT initial segment by expressing Cav1.2 with mouse $\alpha_{1 c} \Delta 1821$ containing alanine substitutions of a.a. 2-5 $\left(\alpha_{1 C} N T-4 A \Delta 1821\right)$ or the TYP motif $\left(\alpha_{1 C} N T-T Y P \Delta 1821\right)$ (Fig. S1, B-D). PKA-CS injection into oocytes expressing $\alpha_{1 C} \Delta 1821$ with intact NT increased $\mathrm{I}_{\mathrm{Ba}}$ by $\sim 70 \%$ ( $\left.p=0.004\right)$. In contrast, oocytes expressing $\alpha_{1 C}$ NT-4A $\Delta 1821$ or $\alpha_{1 C}$ NT-TYP $\Delta 1821$ did not respond to PKA-CS. Thus, as shown previously ${ }^{24}$, the initial segment of the long-NT- $\alpha_{1 C}$, including the first 5 a.a. and the TYP motif, is essential for the Rad-independent PKA regulation of cardiac Cav1.2. 
To further address the role of the NT of $\alpha_{1 c} \Delta 1821$ in Rad-independent vs. Raddependent PKA regulation of Cav1.2, we used NT-truncated- $\alpha_{1 \mathrm{C}} \Delta 1821$ with deletion of the first 20 a.a. $\left(\alpha_{1 C} \Delta 20 \Delta 1821\right.$ ) (see Fig. $1 \mathrm{~A}$ ). We verified that the Rad-independent increase in $\mathrm{I}_{\mathrm{Ba}}$ of $\alpha_{1 c} \Delta 1821$ (34.4 $\left.\pm 7.5 \% ; n=10\right)$ was greatly diminished in $\alpha_{1 c} \Delta 20 \Delta 1821(5 \pm 2 \%, n=16 ; p<0.001$ compared to $\alpha_{1 c} \Delta 1821$ ) (Fig. 3A, B). In contrast, co-expression of Rad resulted in similar $\sim 1.7-2$ fold increases in $\mathrm{I}_{\mathrm{Ba}}$ following cAMP injection when Cav1.2 contained either $\alpha_{1 \mathrm{C}} \Delta 1821$ or $\alpha_{1 C} \Delta 20 \Delta 1821$ ( $\left.p=0.154\right)$. In both constructs, the fold increase in $I_{B a}$ was statistically significant in the presence of Rad, and significantly greater than without Rad (Fig. 3). Thus, Rad-dependent regulation does not require the presence of the NT inhibitory module.

\section{The role of distal C-terminus of $\alpha_{1 c}$ in PKA regulation}

It has been proposed that the cleaved $\mathrm{dCT}$ is a potent autoinhibitory domain that reassociates with the truncated $\alpha_{1 c}$, forming a tight molecular complex ${ }^{8}$ that is essential for PKA regulation of Cav1.2 $2^{23}$. In addition, the cleaved $\mathrm{dCT}$ has been reported to traffic to the nucleus where it serves as a transcription regulator ${ }^{36,37}$. However, in Xenopus oocytes, the presence of $\mathrm{dCT}$ as a separate protein was not required for Rad-independent regulation of Cav1.2- $\Delta 1821^{24}$. Recently, forskolin-induced upregulation of Cav1.2 was demonstrated in HEK293T cells coexpressing Rad and full-length Cav1.2 channels ${ }^{15}$. All in all, the role of $\mathrm{dCT}$ and its truncation in Rad-dependent regulation remains incompletely understood, and it is unknown whether this PKA regulation equally affects full-length and truncated forms of $\alpha_{1 c}$.

To examine the role of dCT in Rad-dependent PKA regulation of Cav1.2, we compared the effect of cAMP on either full length (wt) $\alpha_{1 c}$ or $\alpha_{1 c} \Delta 1821$ channels, in the absence and presence of Rad. We also examined the effect of dCT when coexpressed as a separate protein. $\mathrm{I}_{\mathrm{Ba}}$ is greatly increased by the truncation of $\mathrm{dCT}^{38}$. Therefore, to maintain similar macroscopic currents, we injected different amounts of the channel's subunit RNAs: 1-1.5ng RNA for Cav1.2 containing $\alpha_{1 c} \Delta 1821$, and 1.8-5 ng RNA for Cav1.2 containing wt- $\alpha_{1 c}$. The dCT: $\alpha_{1 c} \Delta 1821$ RNA ratio was 5:1 and Rad: $\beta_{2 b}$ RNA ratios were in the range of 1:3 to 1:1 (which yielded similar increase in $\mathrm{I}_{\mathrm{Ba}}$ when CAMP is injected; see Fig. 1E). As with $\alpha_{1 \mathrm{C}} \Delta 1821$, co-expression of Rad reduced basal currents of Cav1.2 containing wt- $\alpha_{1 C}$, with median $I_{B a}$ of 3.05 $\mu A$ [IQR 2.46-4.73] without Rad and 0.62 $\mu$ A [IQR 0.47-0.73] with Rad ( $p<0.001$; Fig. 4B). 
Injecting CAMP into cells expressing wt- $\alpha_{1 C}$ did not increase $I_{\mathrm{Ba}}$ (actually, a slight reduction of $4 \pm 1.5 \%, n=12$, was observed: Fig. 4A, upper panel; Fig. 4B, C). In contrast, when Rad was coexpressed with wt- $\alpha_{1 c}$, cAMP injection resulted in statistically significant increase of $90 \pm 21 \%$ in $\mathrm{I}_{\mathrm{Ba}}(\mathrm{n}=11)$ (Fig. 4A, lower panel; Fig. 4B, C). Interestingly, in the same experiments, the Rad-dependent cAMP-induced increase in $\mathrm{I}_{\mathrm{Ba}}$ appeared higher in oocytes co-expressing Rad with $\alpha_{1 \mathrm{C}} \Delta 1821: 128 \pm 23 \%, \mathrm{n}=18$. Pairwise comparison of fold increase in $\mathrm{I}_{\mathrm{Ba}}$ for Cav1.2 with wt$\alpha_{1 C}$ vs. $\alpha_{1 c} \Delta 1821$ showed a mildly significant difference, $p=0.04$ (Mann-Whitney test, median 2.06 [IQR 1.77-2.63] for $\alpha_{1 \mathrm{c}} \Delta 1821$ vs. median 1.54 [IQR 1.49-2.04] for wt- $\alpha_{1 \mathrm{C}}$ ). When dCT was co-expressed as a separate protein with $\alpha_{1 c} \Delta 1821$, it did not affect regulation by cAMP even in the presence of Rad ( $p=0.48$ ) (Fig. S2). Thus, unlike Rad-independent regulation, Rad-dependent PKA regulation does not require the cleavage of $\mathrm{dCT}$. Furthermore, the clipped dCT does not appear to play a role in Rad-dependent PKA regulation of $\alpha_{1 \mathrm{c}} \Delta 1821$. However, there appears to be a quantitative difference in the overall cAMP regulation of full-length versus truncated Cav1.2.

\section{Full reconstitution of the $\mathbf{6 1}$ adrenergic receptor regulation of $\mathrm{Ca}_{v} 1.2$}

Despite more than a 3-decade effort, it has not yet been possible to reconstitute the entire adrenergic regulation of cardiac Cav1.2 in a heterologous model. Here we report the reconstitution of the full cascade, starting with activation of $\beta 1-A R$. The initial experiments were conducted with Cav1.2 containing $\alpha_{1 c} \Delta 1821$, using a Rad: $\beta_{2 b}$ RNA ratio of $1: 2$. In the absence of Rad, isoproterenol (Iso; $50 \mu \mathrm{M}$ ), a non-selective $\beta$-AR agonist ${ }^{39}$, did not produce any significant increase in $\mathrm{I}_{\mathrm{Ba}}$ (Fig. 5D, E; Fig. 6). However, co-expression of Rad resulted in a significant increase in $\mathrm{I}_{\mathrm{Ba}}$ following Iso application. Fig. 5A-B shows traces of currents (Fig. 5A) and current-voltage relationship (Fig. 5B) in a representative oocyte. Fig. 5C shows a conductance-voltage curve drawn from 7 oocytes of the same day's experiment. Iso not only increased currents amplitudes, but also caused a $\sim 5 \mathrm{mV}$ hyperpolarization shift in the $\mathrm{V}_{1 / 2}$ for activation, without changing the slope factor (Fig. 5B, lower panel).

In cardiomyocytes, both full-length (wt) and truncated $\alpha_{1 c}$ are present, but it is not known if both isoforms are equally regulated by $\beta 1-A R$. To study $\beta 1-A R$ regulation of Cav1.2 containing wt- $\alpha_{1 c}$, we first titrated Rad: $\beta_{2 b}$, in oocytes that coexpressed $\beta 1-A R$. As for $\alpha_{1 c} \Delta 1821$ 
(see Fig. 1B), we found an inverse correlation between Rad concentration and $I_{\mathrm{Ba}}(\mathrm{r}=-0.85$, $\mathrm{p}=0.002$; Fig. S3). In addition, compared with $\alpha_{1 c} \Delta 1821$, lower Rad: $\beta_{2 b}$ RNA ratios were sufficient to yield a significant increase in currents upon perfusion of Iso (Fig. 5D-E, compare with Fig. 1E). In contrast, in the absence of Rad, there was no increase in currents over time during Iso perfusion (Fig. 5D-E; Fig. 6). Based on the results of Rad titration of Fig. 5, we used Rad: $\beta_{2 b}$ RNA ratio of $1: 3$ to $1: 2$ thereafter for channels containing wt- $\alpha_{1 c}$.

We next systematically compared the effect of Iso on channels containing either wt- $\alpha_{1 C}$ or $\alpha_{1 c} \Delta 1821$, with or without coexpressed $\beta 1-A R$ and Rad (Fig. 6). Without the coexpression of Rad, activation of $\beta 1-A R$ did not produce any increase in $I_{\mathrm{Ba}}$ in either full-length or truncated channel. This result indicates that the Rad-independent pathway is not activated by $\beta 1-A R$ under the conditions used. Moreover, it appears that oocytes do not contain endogenous Rad or similar RGK proteins that are available for the $\beta 1-A R-C a v 1.2$ cascade. Interestingly, when oocytes expressed Rad without the receptor, Iso caused a small increase in $\mathrm{I}_{\mathrm{Ba}}$ : $15 \%$ in $\alpha_{1 c} \Delta 1821$ (which did not reach statistical significance, $p=0.08$ by paired t-test), and $~ 33 \%$ in wt$\alpha_{1 C}(p=0.016)$ (Fig. $\left.6 B, C\right)$. These results corroborate a previous report ${ }^{40}$ suggesting that endogenous $\beta-A R$ is present in some oocyte batches.

In oocytes that expressed $\beta 1-A R$, Rad and Cav1.2, Iso induced a robust increase in $I_{B a}$ (Fig. 6A, B; $p<0.001$ for both $\alpha_{1 C}$ forms). However, comparison of Iso-induced increase in the two $\alpha_{1 C}$ forms revealed a significantly greater effect on the truncated channel than on the fulllength $\alpha_{1 C}\left(147 \%\right.$ vs. $87 \%$ increase in mean $I_{\mathrm{Ba}}$, respectively; median fold increase 2.2 [IQR 1.722.91] versus 1.71 [IQR 1.53-2.07], $p=0.002 ; \mathrm{Fig}$. 6C). These observations are in agreement with the greater effect of CAMP on the truncated channel (Fig. 4) and imply that the dCT does play a role in attenuating the PKA-induced augmentation of Cav1.2 currents, at least when it is not cleaved from $\alpha_{1 c}$.

\section{Reconstitution of the $\beta 2$ adrenergic receptor regulation of $\mathrm{Ca}_{v} 1.2$}

In healthy heart, the distribution of $\beta 2-A R$ is limited to specific parts of the heart (atrium, apex) and mainly to T-tubules within cardiomyocytes, but becomes more widely distributed over the cardiomyocyte surface in failing heart ${ }^{11,41,42} \cdot \beta 2$-AR is also the major $\beta$-AR 
form in the nervous system ${ }^{9}$. We began with titration of $\beta 2-A R$ with Cav1.2 containing $\alpha_{1 c} \Delta 1821$. We expressed the receptors by injecting their RNAs at 50 and $200 \mathrm{pg}$ RNA/oocyte. We also injected higher RNA doses, but above $1 \mathrm{ng} /$ oocyte, cells showed low rate of survival during incubation, and surviving oocytes had high leak currents.

As shown before with cAMP injection (Fig. 1), in oocytes expressing Cav1.2- $\alpha_{1 c} \Delta 1821$, Rad and $\beta 1-A R$, a robust regulation of $I_{B a}$ by Iso was observed (Fig. 7A upper trace, Fig. 7 B, C). Surprisingly, in oocytes of the same batch expressing Cav1.2- $\alpha_{1 \mathrm{C}} \Delta 1821, \beta 2-A R$ and Rad, $I_{\mathrm{Ba}}$ did not respond to Iso (Fig. 7A lower panel, Fig. 7B-C). In some cases, we did observe an increase in $I_{\mathrm{Ba}}$ (e.g. Fig. 8G) but it was very small compared to $\beta 1$-induced Cav1.2 stimulation. To test if the expressed $\beta 2$-AR was functioning well, we used cystic fibrosis transmembrane conductance regulator (CFTR) channel as a control. PKA phosphorylation of CFTR, a chloride channel, activates the channel, leading to an increased outward chloride current ${ }^{43}$. Moreover, CFTR is robustly activated by CAMP and PKA-CS injection in Xenopus oocytes ${ }^{44}$.

Co-expressing CFTR with a range of $\beta 2-A R$ doses (RNA range, 5-50 pg/oocyte) was associated with significantly higher basal chloride currents $\left(\mathrm{I}_{\text {CFTR }}\right)$ compared with cells expressing CFTR alone ( $p<0.001$ ) (Fig. 8B). Iso perfusion did not cause an increase in $\mathrm{I}_{\text {CFTR }}$ at any of the $\beta 2-A R$ doses (Fig. 8A upper panel, Fig. 8C, red circles). This observation implied that CFTR channels may have already been opened by agonist-independent constitutively active $\beta 2-A R s$, thus blunting any response to Iso. Reducing the basal activity of the receptor would result in lower chloride basal currents and may reinstate the activation of $\beta 2$-AR upon Iso perfusion. Propranolol is a non-selective $\beta$ blocker and an inverse agonist, which is known to reduce the constitutive activity of $\beta 2-A R^{45}$. Therefore, we incubated the oocytes for 1-2 hours with $10 \mu \mathrm{M}$ propranolol before starting the recording. Just before the recording, the oocyte was placed in the experimental chamber, voltage clamp was established, and the cell was washed with propranolol-free solution for 2-4 minutes before application of Iso. With propranolol preincubation, we found that Iso induced a robust 1.5-3 fold increase in chloride currents in oocytes expressing CFTR and $\beta 2-A R$ (Fig. 8A lower panel and Fig. 8C, purple circles).

The results of the CFTR experiment strongly supported the possibility that high constitutive activity of $\beta 2-A R$ precluded further effect of Iso, also in the case of Cav1.2. 
Therefore, we employed the propranolol preincubation protocol for Cav1.2- $\alpha_{1 \mathrm{c}} \Delta 1821$, coexpressed with $\beta 2-A R$ and Rad. We measured $I_{\mathrm{Ba}}$ in oocytes not preincubated (black) or preincubated (purple) with propranolol (Fig. 8D). As predicted, preincubation with propranolol resulted in a statistically significant reduction of basal $\mathrm{I}_{\mathrm{Ba}}$ (Fig. 8D). Application of Iso produced only a mild but significant increase in $I_{\mathrm{Ba}}$ without propranolol incubation, by $24 \pm 5 \%$, in this experiment (Fig. 8E, G, H) compared to a significantly greater increase, 3-5 fold, with propranolol pre-incubation (Fig. 8F-H). We conclude that we successfully reconstituted adrenergic regulation of the Cav1.2 channel current as mediated by the two canonical and physiologically-relevant adrenergic receptors.

\section{Discussion}

Numerous biological regulations of ion channels that have been fully reconstituted in heterologous systems accelerated the understanding of their mechanisms and structurefunction relationships. However, the classical adrenergic regulation of cardiac L-type $\mathrm{Ca}^{2+}$ channel remained an unmet challenge for several decades. The recent discovery of the crucial role of $\operatorname{Rad}^{15}$ was a turning point. Here we report, for the first time, the heterologous reconstitution of the full cascade of $\beta$-AR regulation of the cardiac L-type $\mathrm{Ca}^{2+}$ channel, Cav1.2, in Xenopus oocytes, starting with the receptor. Two major advantages of the Xenopus oocyte model are accurate titration of protein expression (by titrated RNA injection), and the ability to co-express a large number of proteins. We utilized the simplicity and robustness of the oocyte expression system to reconstitute the full $\beta$-AR cascade, to address the role of Rad, the relation between Rad-dependent and the previously reported Rad-independent PKA regulation ${ }^{24}$, and to elaborate the role of $\operatorname{Cav} \beta$ and the distal parts of $\mathrm{N}$ - and $\mathrm{C}$-termini of $\alpha_{1 \mathrm{C}}$.

First, we confirmed the CAMP-induced upregulation of $\mathrm{I}_{\mathrm{Ba}}$ in oocytes expressing Cav1.2$\alpha_{1 c} \Delta 1821$ without $\operatorname{Rad}^{24}$ which, in this series of experiments, was $\sim 20 \%$. This is less than the previously reported $30-40 \%{ }^{24}$, probably because here we injected about half the amount of cAMP. Notably, a greater Rad-independent $I_{B a}$ potentiation was attained by injecting purified PKA-CS (Fig. S1).

Next, we scrutinized the role of Rad, initially using CAMP injection to activate the oocyte's endogenous PKA. Liu et al. reported a 1.5-2-fold increase in maximal conductance 
$\left(G_{\max }\right)$ of Cav1.2 containing the full-length $\alpha_{1 C}$ in HEK cells ${ }^{15}$. Here, with $\alpha_{1 \mathrm{C}} \Delta 1821$, titrated

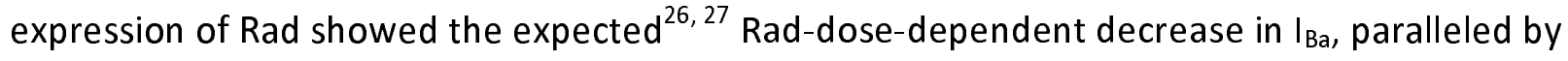
a robust enhancement in cAMP-induced increase in $\mathrm{I}_{\mathrm{Ba}}$, up to $\sim 2.2$-fold (Fig. 1). These results confirm the importance of Rad in PKA regulation and, moreover, suggest that Rad-dependent regulation applies to both full-length and C-terminally-truncated $\alpha_{1 c}$. Importantly, the Radinduced enhancement by cAMP leveled off and occasionally seemed to diminish at higher Rad:Cav1.2 RNA ratios (we expressed all channel subunits in equal weights doses), suggesting that molar excess of Rad may override the PKA-induced abolition of tonic Rad inhibition. Thus, we used the optimal Rad: $\beta_{2 b}$ RNA ratio, 1:3 - 1:1, in all experiments.

We next inquired whether Rad-dependent and Rad-independent regulatory mechanisms are distinct, considering the possibility that the latter was actually the same as Rad-dependent regulation, possibly mediated by an endogenous RGK protein in the oocyte. Our results unequivocally demonstrate that the two modalities are carried out by distinct molecular mechanisms. First, Rad-dependent regulation was fully Cav $\beta$-dependent, as demonstrated ${ }^{15}$. Both Rad inhibition of the basal $I_{\mathrm{Ba}}$, and the Rad-dependent enhancement of cAMP effect critically depended on the presence of coexpressed Cav $\beta$ subunit (full-length or core), and both Rad actions were suppressed by a triple mutation that abolishes Rad-Cav $\beta$ interaction (Fig. 2). In contrast, the Rad-independent cAMP effect was the same with all $\beta_{2 b}$ constructs used, or without coexpressed Cav $\beta$. Second, as shown before ${ }^{24}$, the Rad-independent regulation crucially depended on two cytosolic elements of $\alpha_{1 c}$ : the inhibitory module (initial segment) of the NT (Fig. 3, Fig. S1), and truncation of dCT of $\alpha_{1 C}$ (Fig. 4). In contrast, in the presence of Rad, robust regulation of $\mathrm{I}_{\mathrm{Ba}}$ by CAMP was consistently observed, both after the deletion of the initial NT segment, and in channels containing either full-length or dCT-truncated $\alpha_{1 c}$.

We then reconstituted the full $\beta$-AR cascade with coexpressed $\beta 1-A R$. Activation of $\beta 1-$ $A R$ by Iso caused a $>2$-fold increase in Cav1.2- $\alpha_{1 c} \Delta 1821$ current and the typical hyperpolarizing shift in voltage dependence of activation (Fig. 5, 6), resembling cardiomyocytes ${ }^{16}$. Coexpression of $\mathrm{G}_{\mathrm{s}}$ and adenylyl cyclase was not necessary, suggesting sufficient levels of endogenous proteins. However, expression of Rad was essential; in its absence, no increase in $\mathrm{I}_{\mathrm{Ba}}$ was observed. It is unclear why the Rad-independent regulation of Cav1.2- $\alpha_{1 C} \Delta 1821$ could not be 
produced by activation of $\beta 1-A R$. We consider several possibilities, among them another missing factor needed for this regulation, or a stoichiometry predicament with either the receptor or a downstream protein of the cascade. Unfortunately, expressing high doses or $\beta 1$ $A R, G \alpha_{s}$ or adenylyl cyclase consistently resulted in high oocyte mortality. Whatever the reason, it appeared that, under the conditions of our experiments, $\beta 1-A R$ regulated Cav1.2 only via the Rad-dependent mechanism.

Of particular importance is the observation that, once Rad was present, both dCTtruncated and full length channels were upregulated both by CAMP and by $\beta 1$-AR (Figs. 5,6$)$. This was never clear, and previously controversial ${ }^{18}$. The full-length $\alpha_{1 C}$ is present in the heart and seems even more abundant in neurons ${ }^{9}$. Yet, the mechanism of regulation of neuronal Cav1.2 appears different from that of cardiac; the direct PKA phosphorylation of serine 1928 (located in the dCT) is highly important in neurons ${ }^{46}$, but not in the heart ${ }^{13,47}$. However, in our system the $\beta 1-A R$ activation of full-length $\alpha_{1 c}$-based channels (containing S1928) still required Rad. This indicates that phosphorylation of S1928 alone is not enough. Interestingly, a more detailed examination of the results reveals a potentially important difference: with cAMP, and even more so with $\beta 1-A R$, the overall regulation of the dCT-truncated channel is significantly stronger than of the full-length channel. A more straightforward interpretation would be the contribution of Rad-independent regulation, which is present only in $\alpha_{1 c} \Delta 1821$. However, this is valid only for CAMP-induced regulation; as discussed, $\beta 1$-AR does not appear to initiate the Radindependent pathway. We propose that the $\mathrm{dCT}$ exerts a regulatory control over the Raddependent $\beta$-AR regulation of the channel, via mechanisms that need to be explored.

Finally, we were also able to reconstitute the Cav1.2 enhancement by $\beta 2-A R$ which, like $\beta 1-A R$, acted via the Rad-dependent pathway (Figs. 7, 8). However, there was a significant difference. As demonstrated with both CFTR and Cav1.2, $\beta 2-A R$ constitutively activated the $\mathrm{G}_{\mathrm{s}}{ }^{-}$ PKA pathway, rendering very high basal CFTR and Cav1.2 currents, as revealed by the inverse agonist, propranolol (Fig. 8). The basal Cav1.2 activation was so strong even with very low doses of $\beta 2-A R$ RNA, that pretreatment with propranolol was required to observe upregulation by Iso. Whereas high basal constitutive activity of $\beta 2$-AR is well established ${ }^{45,48,49}$, in the heart, agonist stimulation of $\beta 2$-AR normally increases Cav1.2 currents $^{39,42}$. We assume that, in 
cardiomyocytes, specific mechanisms such as restricted localization ${ }^{9,41,42}$ or additional auxiliary proteins may regulate the basal activity of $\beta 2-A R$.

In summary, we reconstituted the $\beta 1-A R$ and $\beta 2-A R$ regulation of Cav1.2 in the Xenopus oocyte model system. We demonstrate a robust ( 2 -fold) enhancement of $\mathrm{Ca}^{2+}$ channel currents, by cAMP and Iso, in a Rad-dependent manner, and confirm the existence of a less prominent, separate, Rad-independent PKA regulation of Cav1.2 ( $30 \%$ increase in Cav1.2 current). Our reconstituted system replicates the known basic features of $\beta$-AR regulation of cardiac Cav1.2 and reveals novel molecular details, including the consequences of proteolytic processing of $\alpha_{1 C}$ with respect to $\beta$-AR regulation, the differential roles of Cav $\beta$ subunit and of the $\mathrm{N}$ - and $\mathrm{C}$-termini of $\alpha_{1 \mathrm{C}}$ in the Rad-dependent and Rad-independent regulation, and the differences in channel regulation by $\beta 1-A R$ and $\beta 2-A R$. The reconstitution of the basic cascade will enable further investigation of the mechanisms of Rad action and additional auxiliary proteins implicated in macromolecular complexes involved in $\beta$-AR regulation of Cav1.2, such as A-kinase anchoring proteins, phosphatases and phosphodiesterases, and others ${ }^{50}$. It may also be instrumental in identifying potential targets for therapeutic modulation of $\beta$-adrenergic regulation in heart and other tissues.

\section{References}

1. Bers DM. Calcium cycling and signaling in cardiac myocytes. Annu Rev Physiol. 2008;70:23-49. 10.1146/annurev.physiol.70.113006.100455

2. Catterall WA. Regulation of cardiac calcium channels in the fight-or-flight response. Curr Mol Pharmacol. 2015;8:12-21. 10.2174/1874467208666150507103417

3. Best JM and Kamp TJ. Different subcellular populations of L-type $\mathrm{Ca}^{2+}$ channels exhibit unique regulation and functional roles in cardiomyocytes. J Mol Cell Cardiol. 2012;52:376-87. 10.1016/j.yjmcc.2011.08.014

4. Hofmann F, Flockerzi V, Kahl S and Wegener JW. L-type Cav1.2 calcium channels: from in vitro findings to in vivo function. Physiol Rev. 2014;94:303-26. 10.1152/physrev.00016.2013

5. Dolphin AC. Voltage-gated calcium channels and their auxiliary subunits: physiology and pathophysiology and pharmacology. J Physiol. 2016;594:5369-90. 10.1113/JP272262 
6. Gao T, Puri TS, Gerhardstein BL, Chien AJ, Green RD and Hosey MM. Identification and subcellular localization of the subunits of L-type calcium channels and adenylyl cyclase in cardiac myocytes. J Biol Chem. 1997;272:19401-7. 10.1074/jbc.272.31.19401

7. Katchman A, Yang L, Zakharov SI, Kushner J, Abrams J, Chen BX, Liu G, Pitt GS, Colecraft $\mathrm{HM}$ and Marx SO. Proteolytic cleavage and PKA phosphorylation of $\alpha 1 \mathrm{C}$ subunit are not required for adrenergic regulation of Cav1.2 in the heart. Proc Natl Acad Sci U SA.

2017;114:9194-9199. 10.1073/pnas.1706054114

8. Hulme JT, Yarov-Yarovoy V, Lin TW, Scheuer T and Catterall WA. Autoinhibitory control of the Cav1.2 channel by its proteolytically processed distal C-terminal domain. J Physiol. 2006;576:87-102. 10.1113/jphysiol.2006.111799

9. Dai S, Hall DD and Hell JW. Supramolecular assemblies and localized regulation of voltage-gated ion channels. Physiol Rev. 2009;89:411-52. 10.1152/physrev.00029.2007 10. Xiao RP, Zhu W, Zheng M, Chakir K, Bond R, Lakatta EG and Cheng H. Subtype-specific $\beta$ adrenoceptor signaling pathways in the heart and their potential clinical implications. Trends Pharmacol Sci. 2004;25:358-65. 10.1016/j.tips.2004.05.007

11. Nikolaev VO, Moshkov A, Lyon AR, Miragoli M, Novak P, Paur H, Lohse MJ, Korchev YE, Harding SE and Gorelik J. $\beta 2$-adrenergic receptor redistribution in heart failure changes CAMP compartmentation. Science. 2010;327:1653-7. 10.1126/science.1185988

12. Xiao RP, JiX and Lakatta EG. Functional coupling of the $\beta 2$-adrenoceptor to a pertussis toxin-sensitive G protein in cardiac myocytes. Mol Pharmacol. 1995;47:322-9.

13. Lemke $T$, Welling A, Christel CJ, Blaich A, Bernhard D, Lenhardt $P$, Hofmann $F$ and Moosmang S. Unchanged $\beta$-adrenergic stimulation of cardiac L-type calcium channels in Cav1.2 phosphorylation site S1928A mutant mice. J Biol Chem. 2008;283:34738-44.

\subsection{4/jbc.M804981200}

14. Yang L, Katchman A, Samad T, Morrow J, Weinberg R and Marx SO. $\beta$-adrenergic regulation of the L-type $\mathrm{Ca}^{2+}$ channel does not require phosphorylation of $\alpha_{1 C}$ Ser1700. Circ Res. 2013;113:871-80. 10.1161/CIRCRESAHA.113.301926

15. Liu G, Papa A, Katchman AN, Zakharov SI, Roybal D, Hennessey JA, Kushner J, Yang L, Chen BX, Kushnir A, Dangas K, Gygi SP, Pitt GS, Colecraft HM, Ben-Johny M, Kalocsay M and 
Marx SO. Mechanism of adrenergic Cav1.2 stimulation revealed by proximity proteomics. Nature. 2020;577:695-700. 10.1038/s41586-020-1947-z

16. Miriyala J, Nguyen T, Yue DT and Colecraft HM. Role of Cav $\beta$ subunits, and lack of functional reserve, in protein kinase A modulation of cardiac Cav1.2 channels. Circ Res. 2008;102:e54-64. 10.1161/CIRCRESAHA.108.171736

17. Roybal D, Hennessey JA and Marx SO. The quest to identify the mechanism underlying adrenergic regulation of cardiac $\mathrm{Ca}^{2+}$ channels. Channels (Austin). 2020;14:123-131.

10.1080/19336950.2020.1740502

18. Weiss S, Oz S, Benmocha A and Dascal N. Regulation of cardiac L-type Ca ${ }^{2+}$ channel Cav1.2 via the $\beta$-adrenergic-cAMP-protein kinase A pathway: old dogmas, advances, and new uncertainties. Circ Res. 2013;113:617-31. 10.1161/CIRCRESAHA.113.301781

19. Singer-Lahat D, Lotan I, Biel M, Flockerzi V, Hofmann F and Dascal N. Cardiac calcium channels expressed in Xenopus oocytes are modulated by dephosphorylation but not by cAMPdependent phosphorylation. Receptors Channels. 1994;2:215-26.

20. Charnet $\mathrm{P}$, Lory $\mathrm{P}$, Bourinet $\mathrm{E}$, Collin T and Nargeot J. CAMP-dependent phosphorylation of the cardiac L-type Ca channel: a missing link? Biochimie. 1995;77:957-62. 10.1016/03009084(95)80008-5

21. Perez-Reyes E, Yuan W, Wei X and Bers DM. Regulation of the cloned L-type cardiac calcium channel by cyclic-AMP-dependent protein kinase. FEBS Lett. 1994;342:119-23. 10.1016/0014-5793(94)80484-2

22. Dascal N, Snutch TP, Lubbert H, Davidson N and Lester HA. Expression and modulation of voltage-gated calcium channels after RNA injection in Xenopus oocytes. Science. 1986;231:1147-50. 10.1126/science.2418503

23. Fuller MD, Emrick MA, Sadilek M, Scheuer T and Catterall WA. Molecular mechanism of calcium channel regulation in the fight-or-flight response. Sci Signal. 2010;3:ra70. 10.1126/scisignal. 2001152

24. Oz S, Pankonien I, Belkacemi A, Flockerzi V, Klussmann E, Haase H and Dascal N. Protein kinase A regulates C-terminally truncated Cav1.2 in Xenopus oocytes: roles of $\mathrm{N}$ - and C-termini of the $\alpha_{1 C}$ subunit. J Physiol. 2017;595:3181-3202. 10.1113/JP274015 
25. Correll RN, Pang C, Niedowicz DM, Finlin BS and Andres DA. The RGK family of GTPbinding proteins: regulators of voltage-dependent calcium channels and cytoskeleton remodeling. Cellular signalling. 2008;20:292-300. 10.1016/j.cellsig.2007.10.028

26. Finlin BS, Crump SM, Satin J and Andres DA. Regulation of voltage-gated calcium channel activity by the Rem and Rad GTPases. Proc Natl Acad Sci USA. 2003;100:14469-74. 10.1073/pnas. 2437756100

27. Yang T and Colecraft HM. Regulation of voltage-dependent calcium channels by RGK proteins. Biochimica et biophysica acta. 2013;1828:1644-54. 10.1016/j.bbamem.2012.10.005 28. Manning JR, Yin G, Kaminski CN, Magyar J, Feng HZ, Penn J, Sievert G, Thompson K, Jin JP, Andres DA and Satin J. Rad GTPase deletion increases L-type calcium channel current leading to increased cardiac contraction. J Am Heart Assoc. 2013;2:e000459. 10.1161/JAHA.113.000459

29. Hullin R, Singer-Lahat D, Freichel M, Biel M, Dascal N, Hofmann F and Flockerzi V. Calcium channel $\beta$ subunit heterogeneity: functional expression of cloned cDNA from heart, aorta and brain. EMBO J. 1992;11:885-90.

30. Opatowsky Y, Chen CC, Campbell KP and Hirsch JA. Structural analysis of the voltagedependent calcium channel $\beta$ subunit functional core and its complex with the $\alpha 1$ interaction domain. Neuron. 2004;42:387-99. 10.1016/s0896-6273(04)00250-8

31. Yang T, Puckerin A and Colecraft HM. Distinct RGK GTPases differentially use $\alpha 1-$ and auxiliary $\beta$-binding-dependent mechanisms to inhibit Cav1.2/Cav2.2 channels. PLoS One. 2012;7:e37079. 10.1371/journal.pone.0037079

32. Kanevsky $\mathrm{N}$ and Dascal $\mathrm{N}$. Regulation of maximal open probability is a separable function of Cav $\beta$ subunit in L-type $\mathrm{Ca}^{2+}$ channel, dependent on $\mathrm{NH}_{2}$ terminus of $\alpha_{1 \mathrm{C}}(\mathrm{Cav} 1.2 \alpha)$. J Gen Physiol. 2006;128:15-36. 10.1085/jgp.200609485

33. Sasson Y, Navon-Perry L, Huppert D and Hirsch JA. RGK family G-domain:GTP analog complex structures and nucleotide-binding properties. Journal of molecular biology. 2011;413:372-89. 10.1016/j.jmb.2011.08.017

34. Blumenstein $\mathrm{Y}$, Kanevsky N, Sahar G, Barzilai R, Ivanina T and Dascal N. A novel long-Nterminus isoform of human L-type $\mathrm{Ca}^{2+}$ channel is up-regulated by protein kinase C. J Biol Chem. 2002;277:3419-3423. 
35. Béguin P. RGK small GTP-binding proteins interact with the nucleotide kinase domain of $\mathrm{Ca}^{2+}$-channel $\beta$-subunits via an uncommon effector binding domain. $J$ Biol Chem. 2007;282:11509-20. 10.1074/jbc.M606423200

36. Gomez-Ospina N, Tsuruta F, Barreto-Chang O, Hu L and Dolmetsch R. The C terminus of the L-type voltage-gated calcium channel Cav1.2 encodes a transcription factor. Cell. 2006;127:591-606. 10.1016/j.cell.2006.10.017

37. Schroder E, Byse $M$ and Satin J. L-type calcium channel $C$ terminus autoregulates transcription. Circ Res. 2009;104:1373-81. 10.1161/CIRCRESAHA.108.191387

38. Wei X, Neely A, Lacerda AE, Olcese R, Stefani E, Perez Reyes E and Birnbaumer L. Modification of $\mathrm{Ca}^{2+}$ channel activity by deletions at the carboxyl terminus of the cardiac $\alpha_{1}$ subunit. J Biol Chem. 1994;269:1635-40.

39. Skeberdis VA, Jurevicius J and Fischmeister a R. $\beta 2$ adrenergic activation of L-type $\mathrm{Ca}^{++}$ current in cardiac myocytes. J Pharmacol Exp Ther. 1997;283:452-61.

40. Kusano K, Miledi R and Stinnakre J. Cholinergic and catecholaminergic receptors in the Xenopus oocyte membrane. J Physiol. 1982;328:143-70. 10.1113/jphysiol.1982.sp014257

41. Wright PT, Bhogal NK, Diakonov I, Pannell LMK, Perera RK, Bork NI, Schobesberger S, Lucarelli C, Faggian G, Alvarez-Laviada A, Zaccolo M, Kamp TJ, Balijepalli RC, Lyon AR, Harding SE, Nikolaev VO and Gorelik J. Cardiomyocyte membrane structure and cAMP compartmentation produce anatomical variation in $\beta_{2} A R$-cAMP responsiveness in murine hearts. Cell reports. 2018;23:459-469. 10.1016/j.celrep.2018.03.053

42. Bryant SM, Kong CHT, Cannell MB, Orchard CH and James AF. Loss of caveolin-3dependent regulation of $\mathrm{I}_{\mathrm{Ca}}$ in rat ventricular myocytes in heart failure. Am J Physiol. 2018;314:H521-h529. 10.1152/ajpheart.00458.2017

43. Welsh MJ, Anderson MP, Rich DP, Berger HA, Denning GM, Ostedgaard LS, Sheppard DN, Cheng SH, Gregory RJ and Smith AE. Cystic fibrosis transmembrane conductance regulator: a chloride channel with novel regulation. Neuron. 1992;8:821-9.

http://dx.doi.org/10.1016/0896-6273(92)90196-K

44. Bear CE, Duguay F, Naismith AL, Kartner N, Hanrahan JW and Riordan JR. Cl- channel activity in Xenopus oocytes expressing the cystic fibrosis gene. J Biol Chem. 1991;266:19142-5. 
45. Chidiac $P$, Hebert TE, Valiquette $M$, Dennis $M$ and Bouvier $M$. Inverse agonist activity of $\beta$-adrenergic antagonists. Mol Pharmacol. 1994;45:490-9.

46. Qian H, Patriarchi T, Price JL, Matt L, Lee B, Nieves-Cintron M, Buonarati OR, Chowdhury D, Nanou E, Nystoriak MA, Catterall WA, Poomvanicha M, Hofmann F, Navedo MF and Hell JW. Phosphorylation of Ser1928 mediates the enhanced activity of the L-type $\mathrm{Ca}^{2+}$ channel Cav1.2 by the $\beta 2$-adrenergic receptor in neurons. Sci Signal. 2017;10. 10.1126/scisignal.aaf9659

47. Ganesan AN, Maack C, Johns DC, Sidor A and O'Rourke B. $\beta$-adrenergic stimulation of Ltype Ca2+ channels in cardiac myocytes requires the distal carboxyl terminus of $\alpha 1 \mathrm{C}$ but not serine 1928. Circ Res. 2006;98:e11-8. 10.1161/01.RES.0000202692.23001.e2

48. Chakir K, Xiang Y, Yang D, Zhang SJ, Cheng H, Kobilka BK and Xiao RP. The third intracellular loop and the carboxyl terminus of $\beta 2$-adrenergic receptor confer spontaneous activity of the receptor. Mol Pharmacol. 2003;64:1048-58. 10.1124/mol.64.5.1048

49. Zhang SJ, Cheng H, Zhou YY, Wang DJ, Zhu W, Ziman B, Spurgoen H, Lefkowitz RJ, Lakatta EG, Koch WJ and Xiao RP. Inhibition of spontaneous $\beta 2$-adrenergic activation rescues $\beta 1$-adrenergic contractile response in cardiomyocytes overexpressing $\beta 2$-adrenoceptor. J Biol Chem. 2000;275:21773-9. 10.1074/jbc.M909484199

50. Pallien T and Klussmann E. New aspects in cardiac L-type $\mathrm{Ca}^{2+}$ channel regulation. Biochem Soc Trans. 2020;48:39-49. 10.1042/bst20190229

\section{Sources of Funding}

This research was supported by the German-Israeli Science Foundation (GIF grant I-1452203.13/2018) to N.D., E.K., V.F. and S.W., The Gessner Fund to M.K. and N.D., the Deutsche Forschungsgemeinschaft (German Research Foundation, DFG KL1415/7-1, to E.K.), the Deutsche Forschungsgemeinschaft grant to A.B. and V.F. (SFB 894, TP A3), the Israel Science Foundation grants $1519 / 12$ and $1500 / 16$ to J.H., and a Seymour-Fefer grant to M.K. M.K. was supported in part by a scholarship from Alrov Foundation. S.S. was supported in part by a scholarship from the Prajs-Drimmer Institute at Tel Aviv University. 
bioRxiv preprint doi: https://doi.org/10.1101/2020.11.30.403964; this version posted December 1, 2020. The copyright holder for this preprint (which was not certified by peer review) is the author/funder. All rights reserved. No reuse allowed without permission.

\section{Disclosures}

All authors report no potential or existing conflict of interest to disclose. 


\section{Figure legends}

\section{Figure 1.}

Figure 1. cAMP regulation of Cav1.2 is greatly enhanced by co-expression of Rad. A, The cardiac Cav1.2 and Rad. The $\alpha_{1 c}$ and $\alpha 2 \delta$ subunits are shown schematically, along with structures of $\beta_{2 b}$ (PDB:1T3S) ${ }^{30}$ and Rad (PDB: 3Q72) ${ }^{33}$. In $\alpha_{1 c}$, the four homologous transmembrane domains are numbered I to IV. The constructs used here were based on rabbit or mouse long-NT isoforms, containing the 46 a.a.-long initial segment encoded by exon $1 a^{34}$ and either full-length $C T$, or, in $\alpha_{1 c} \Delta 1821$, the $\alpha_{1 c}$ was truncated at a.a. 1821 , close to the naturally occurring truncation site (red cross mark) in cardiac $\alpha_{1 c}$, a.a. $1800^{7}$. $\beta_{2 b}$ binds to the cytosolic loop connecting domains I and II. When recruited to the plasma membrane, Rad interferes with the interaction between $\beta$ and $\alpha_{1 C}$ subunits. B-F, examples and summaries for Cav1,2- $\alpha_{1 C} \Delta 1821$. B, Rad reduces the $\mathrm{Ba}^{2+}$ current of Cav1.2- $\alpha_{1 \mathrm{C}} \Delta 1821$ in a dose-dependent manner. The channel was expressed in full subunit composition: $\alpha_{1 c} \Delta 1821, \beta_{2 b}$ and $\alpha 2 \delta$ (1.5 ng RNA of each subunit). $I_{\mathrm{Ba}}$ decreased with increasing doses of Rad RNA (Pearson correlation, $r=-$ $0.82, p=0.023$ ). Each point represents mean \pm SEM from 7 to 10 oocytes ( $N=1$ experiment). The linear regression line was drawn for non-zero doses of Rad. C, Rad enhances the CAMP-induced increase in $\mathrm{I}_{\mathrm{Ba}}$. Figures are diary plots showing the time course of change in $\mathrm{I}_{\mathrm{Ba}}$ (normalized to initial $I_{\mathrm{Ba}}$ ), before and after the intracellular injection of CAMP in a representative cell. Insets show current records at $+20 \mathrm{mV}$ before (black trace) and 10 min after cAMP injection (red trace), without Rad in the upper panel and with Rad in the lower panel. D, "before-after" plots of cAMP-induced changes in $I_{\mathrm{Ba}}$ in individual cells injected with increasing ratio of Rad: $\beta_{2 b}$ expression. Empty symbols - before cAMP; red-filled - after cAMP. RNA ratios (by weight, w/w) of Rad and $\beta_{2 b}$ were varied in 1:20 to 2:1 range. $N=3$ experiments; statistics: paired t-test. $E$, cAMP-induced increase in $I_{\mathrm{Ba}}$ depends on relative expression levels of Rad and $\beta_{2 b}$. Each symbol represents fold increase in $\mathrm{I}_{\mathrm{Ba}}$ induced by cAMP injection in an individual cell, at different Rad: $\beta_{2 b}$ RNA ratios. Here and in the following figures, box plots show 25-75 percentiles, whiskers show the 5/95 percentiles, the black and the red horizontal lines within the boxes are the median and mean, respectively. At all Rad: $\beta_{2 b}$ RNA ratios except $1: 20$, the cAMP-induced 
increase in $I_{\mathrm{Ba}}$ was significantly greater than without Rad (Kruskal-Wallis One-Way ANOVA on ranks). F, summary of the effect of cAMP in 10 experiments without Rad and with Rad at 1:2 and 1:1 Rad: $\beta_{2 b}$ RNA ratios (pooled). Number of cells is shown within the bars. Statistics: MannWhitney test.

\section{Figure 2.}

Figure 2. Separation of Rad-dependent and Rad-independent PKA regulation of $\alpha_{1 \mathrm{c}}$ : the role of $C_{v} \beta$. A, Schematic representation of $C_{a} \beta$ subunit variants used in this study. The wild-type $\left(\beta_{2 b}-w t\right)$ protein is 632 a.a. long. $\beta$-core is truncated at a.a. 424; the linker amino acids 138-202 were removed $^{30}$. The $\beta$-core sDA $_{3 \mathrm{~A}}$ is the $\beta$-core with three aspartate-to-alanine mutations, D244A/D320A/D322A, that does not bind Rad.. B-D, the presence of the $\beta$ subunit and its ability to bind Rad are crucial for Rad-dependent, but not for Rad-independent, cAMP regulation of Cav1.2. Rad: $\beta_{2 b}$ RNA ratio was $1: 1 . N=1$ experiment. $B$, Diary plots of cAMP-induced changes in $I_{B a}$ following injection of CAMP in representative cells expressing $\alpha_{1 C} \Delta 1821, \alpha 2 \delta$, and the indicated variant of $\beta_{2 b}$ (or no $\beta$ at all), with or without Rad. Insets show current records at +20 $\mathrm{mV}$ before (black trace) and $10 \mathrm{~min}$ after cAMP injection (red trace). C, "Before-after" plots of cAMP-induced changes in $I_{B a}$ in individual cells injected with various Cav $\beta$ subunits co-expressed with and without Rad. $\mathrm{N}=1$ experiment; statistics: paired t-test. $\mathbf{D}$, Fold change in $\mathrm{I}_{\mathrm{Ba}}$ after CAMP injection. Data show the fold increase with Rad co-expression (inverted triangles) and without Rad (circles). Mann-Whitney Rank Sum test was used to compare groups with and without Rad (except the groups with $\beta$-core 3 DA where normality was satisfied, and t-test was used).

\section{Figure 3.}

Figure 3. Separation of Rad-dependent and Rad-independent PKA regulation of $\alpha_{1 c}$ : the role of $\mathrm{N}$-terminal initial segment of $\boldsymbol{\alpha}_{1 \mathrm{C}}$. A, "before-after" plots of CAMP-induced changes in $\mathrm{I}_{\mathrm{Ba}}$ in individual cells expressing Cav1.2 containing $\alpha_{1 c} \Delta 1821$ (black) and $\alpha_{1 C} \Delta 20 \Delta 1821$ (red) lacking the first 20 a.a. of the $\mathrm{N}$-terminus. $\alpha 2 \delta$ and $\beta_{2 b}$ were co-expressed in all cases, without or with Rad (circles and inverted triangles, respectively). N=3 experiments; statistics: paired t-test. B, Fold change in $\mathrm{I}_{\mathrm{Ba}}$ after CAMP injection (summary of experiments shown in A). The black and the 
red horizontal lines within the boxes are the median and mean, respectively. Pairwise comparison with and without Rad was done using the Mann-Whitney Rank Sum test.

\section{Figure 4.}

Figure 4. Separation of Rad-dependent and Rad-independent PKA regulation of $\alpha_{1 \mathrm{c}}$ : the role of the distal C-terminus of $\boldsymbol{\alpha}_{1 \mathrm{c}}$. A, Diary plots of cAMP-induced changes in $\mathrm{I}_{\mathrm{Ba}}$ in representative cells expressing the full length $\alpha_{1 C}\left(\alpha_{1 c}\right.$ WT), $\alpha 2 \delta$ and $\beta_{2 b}$, without Rad (upper panel) or with Rad (lower panel). B, "before-after" plots of CAMP-induced changes in $\mathrm{I}_{\mathrm{Ba}}$ in individual cells expressing wt- $\alpha_{1 c}$ with or without Rad co-expression, or $\alpha_{1 c} \Delta 1821$ and Rad. $\alpha 2 \delta$ and $\beta_{2 b}$ were present in all cases. Rad: $\beta_{2 b}$ RNA ratio was $1: 2$ or $1: 1$. Statistics: paired t-test. $N=3$ experiments. $\mathrm{n}=12,11$ and 18 oocytes, from left to right. $\mathrm{C}$, Fold change in $\mathrm{I}_{\mathrm{Ba}}$ at $+20 \mathrm{mV}$ after cAMP injection (summary of data shown in A, B). The black and the red horizontal lines within the boxes are the median and mean, respectively. Statistics: Kruskal-Wallis One-Way ANOVA on ranks.

\section{Figure 5.}

Figure 5. Full reconstitution of the $\beta 1-A R$ regulation of $\alpha_{1 C}$ A-C, $\beta 1-A R$ regulation of Cav1.2. Oocytes were injected with RNA of $\alpha_{1 c} \Delta 1821, \alpha 2 \delta, \beta_{2 b}$, Rad and $\beta 1-A R$. A, Ba ${ }^{2+}$ currents (upper panels) before (left) and after (right) perfusion of $50 \mu \mathrm{M}$ isoproterenol (Iso) in a representative cell. The voltage protocol is illustrated in the lower panel; $\mathrm{I}_{\mathrm{Ba}}$ was elicited by 20 -ms voltage steps given every $10 \mathrm{~s}$ from a holding potential of $-80 \mathrm{mV}$ in $10 \mathrm{mV}$ increments. The currents shown are net $\mathrm{I}_{\mathrm{Ba}}$ derived by subtraction of the residual currents recorded with the same protocols after applying $200 \mu \mathrm{M} \mathrm{Cd}^{2+}$ (not shown). Since full capacity compensation in oocytes was not achievable, the currents during the first $\sim 2 \mathrm{~ms}$ (the duration of capacity transient) were blanked out. B, Top, representative I-V curve before (black) and after (red) addition of Iso. Bottom, parameters of Boltzmann fit of I-V curves in 7 oocytes, before and after Iso. C, Conductancevoltage (G-V) curves of Cav1.2- $\alpha_{1 c} \Delta 1821$ co-expressed with Rad and $\beta 1-A R$ averaged from oocytes of a representative batch ( $n=7$ oocytes, $N=1$ experiment) before and after Iso. The curves were drawn using the Boltzmann equation using average $V_{1 / 2}$ and $K_{a}$ obtained from the fits of I-V curves in individual oocytes (see Methods). Parameters used were: $V_{1 / 2}=8.7 \mathrm{mV}$, 
$\mathrm{K}_{\mathrm{a}}=6.8 \mathrm{mV}$ before Iso; $\mathrm{V}_{1 / 2}=3.8 \mathrm{mV}, \mathrm{K}_{\mathrm{a}}=6.6 \mathrm{mV}$ after Iso. $\mathrm{D}-\mathrm{E}, \beta 1-\mathrm{AR}$ regulation of Cav1.2 containing the wt- $\alpha_{1 C}$ in the presence of increasing doses of Rad. Oocytes were injected with RNAs of wt- $\alpha_{1 c}, \alpha 2 \delta, \beta_{2 b}, \beta 1 A R$, and the indicated doses of Rad RNA. D, "before-after" plots of Iso-induced changes in $\mathrm{I}_{\mathrm{Ba}}$ in individual cells injected with increasing doses of Rad RNA. N=1 experiment; statistics: paired t-test. $E$, Fold change increase in $I_{\mathrm{Ba}}$ caused by Iso as a function of Rad: $\beta_{2 b}$ RNA ratio. $N=1$ experiment. Statistics: One Way ANOVA.

\section{Figure 6.}

Figure 6. $\beta 1-A R$ regulation of full-length and truncated $\boldsymbol{\alpha}_{1 \mathrm{1C}} . \mathrm{A}$, Representative diary plots of $\mathrm{I}_{\mathrm{Ba}}$ in oocytes expressing wt (full-length) $\alpha_{1 \mathrm{C}}\left(\mathrm{Ca}_{\vee} 1.2-\alpha_{1 \mathrm{C}}\right)$ or Cav1.2- $\alpha_{1 \mathrm{C}} \Delta 1821$ channels (lower and upper panels, respectively) with or without Rad and $\beta 1-A R$, and the response to $50 \mu \mathrm{M}$ Iso. $\beta_{2 b}$ and $\alpha 2 \delta$ were coexpressed in all cases. B, "Before-after" plots of Iso-induced changes in $\mathrm{I}_{\mathrm{Ba}}$ in individual cells. The Rad: $\beta 2 b$ ratio in oocytes expressing wt Cav1.2 (blue symbols) or Cav1.2 1821 (black symbols) was 1:3 and 1:2, respectively. Lower ratio was used in cells expressing wt- $\alpha_{1 C}$ to allow higher basal currents. $\mathrm{N}=3$; statistics: paired t-test. $\mathrm{C}$, Rad is essential for the Iso-induced increase in $\mathrm{I}_{\mathrm{Ba}}$. $\mathrm{N}=3$ experiments. Statistical analysis was performed separately for wt Cav1.2- $\alpha_{1 C}$ and Cav1.2- $\alpha_{1 c} \Delta 1821$ with ANOVA on ranks $(p<0.001)$. In addition, Mann-Whitney Rank Sum test was used to compare the last two groups, wt Cav1.2 vs. Cav1.2 1821 with Rad and $\beta 1-A R(p=0.002)$. For wt Cav1.2 with Rad and $\beta 1-A R$, there was no significant difference from the group without $\beta 1-A R$ but with Rad ( $p=0.076$, One-Way ANOVA on ranks, Dunnett's test).

\section{Figure 7.}

Figure 7. Comparison of $\beta 1$ and $\beta 2$ adrenergic regulation of Cav1.2. $A$, Representative diary plots of $I_{B a}$ showing the effect of Iso on Cav1.2- $\alpha_{1 C} \Delta 1821$ in oocytes co-expressing $\beta 1-A R$ (upper panel) or $\beta 2-A R$ (lower panel). Insets show $\mathrm{I}_{\mathrm{Ba}}$ at $+20 \mathrm{mV}$ before and 9-10 min after the addition of Iso. RNAs of both receptors were made on the template of cDNAs inserted into the pGEM-HJ vector. B, "Before-after" plots of Iso-induced changes in $\mathrm{I}_{\mathrm{Ba}}$ in individual cells co-expressing Cav1.2- $\alpha_{1 c} \Delta 1821$ and Rad, without any receptor or with either $\beta 1-A R$ or $\beta 2-A R$. Data are shown 
for receptors RNAs concentrations of 0,50 and 200 pg RNA/oocyte. Rad: $\beta_{2 b}$ RNA ratio was 1:2. $\mathrm{N}=1$ experiment; statistics: paired t-test. $\mathrm{C}$, Fold change in $\mathrm{I}_{\mathrm{Ba}}$ induced by Iso (summary of data shown in A and B). Statistics: One Way ANOVA followed by Dunnett's test.

\section{Figure 8.}

\section{Figure 8. Reconstitution of the $\beta 2$ adrenergic receptor regulation of CFTR and $\alpha_{1 C}$. A,}

Preincubation with propranolol is needed to reinstate the adrenergic regulation of CFTR by $\beta 2$ AR. Oocytes expressed CFTR and $\beta 2-A R(1$ and 0.005-0.05 ng/oocyte, respectively). CFTR chloride currents were recorded in gap free mode at $-80 \mathrm{mV} .50 \mu \mathrm{m}$ Iso was applied after stabilization of the current (about 2 min after start of record). Upper panel shows a representative record without propranolol incubation. The lower panel shows a representative oocyte preincubated in $10 \mu \mathrm{M}$ propranolol for 60-120 min, then transferred to the experimental chamber, voltage clamped (start of record) and perfused with propranolol-free solution for about 2 min prior to the addition of Iso. B, Basal chloride currents in oocytes coexpressing CFTR channel (1 ng RNA/oocyte) and $\beta 2-A R$ (increasing doses of RNA). Oocytes expressing CFTR alone had statistically significant lower basal currents than oocytes coexpressing $\beta 2-A R$ ( $N=1$ experiment; statistics: $p<0.001$, Kruskal-Wallis One Way ANOVA on Ranks) C, Fold change in chloride current by Iso. The plot compares the effects of $50 \mu \mathrm{M}$ Iso in oocytes injected with the indicated doses of $\beta 2$-AR RNA, without (red symbols) or with (purple symbols) propranolol preincubation. $\mathrm{N}=1$ experiment. Statistics: Mann-Whitney Rank Sum Test. D, Comparison of basal $\mathrm{I}_{\mathrm{Ba}}$ in oocytes co-expressing Cav1.2- $\alpha_{1 \mathrm{C}} \Delta 1821$, Rad and $\beta 2-\mathrm{AR}$, which were incubated without propranolol (black) or with $10 \mu \mathrm{M}$ propranolol (blue). $\mathrm{I}_{\mathrm{Ba}}$ was recorded at peak current of $+20 \mathrm{mV}$. Oocytes were preincubated with $10 \mu \mathrm{M}$ propranolol for $60-120 \mathrm{~min}$, then transferred to the experimental chamber, voltage clamped to $+20 \mathrm{mV}$ and perfused with propranolol-free $40 \mathrm{mM} \mathrm{Ba}^{2+}$ solution until stabilization of the current for $2 \mathrm{~min}$. Propranolol washout lasted approximately 1-2 minutes. Oocytes co-expressed $\alpha_{1 c} \Delta 1821, \beta_{2 b}, \alpha 2 \delta$, Rad (Rad: $\beta 2_{b}$ RNA ratio was 0.5 ). N=1; statistics: t-test. E-F, Representative diary plots of oocytes coexpressing $\alpha_{1 c} \Delta 1821$, Rad and $\beta 2-A R$ without (left) and with (right) preincubation with propranolol. G, "Before-after" plots of Iso-induced changes in $\mathrm{I}_{\mathrm{Ba}}$ in individual cells. Blue 
symbols represent data form oocytes preincubated with propranolol, black - without

preincubation. $\mathrm{N}=1$; statistics: paired t-test. $\mathbf{H}$, Fold increase in $\mathrm{I}_{\mathrm{Ba}}$ induced by Iso (summary of data shown in G). Oocytes preincubated with propranolol (blue) had statistically significant higher fold increase. $\mathrm{N}=1$; statistics: Kruskal-Wallis One Way ANOVA on Ranks followed by Dunnett's test. 


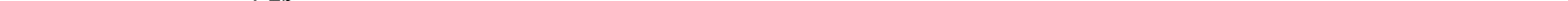


$\beta_{2 b}$-core

$\beta_{2 b}$-core $3 D A$

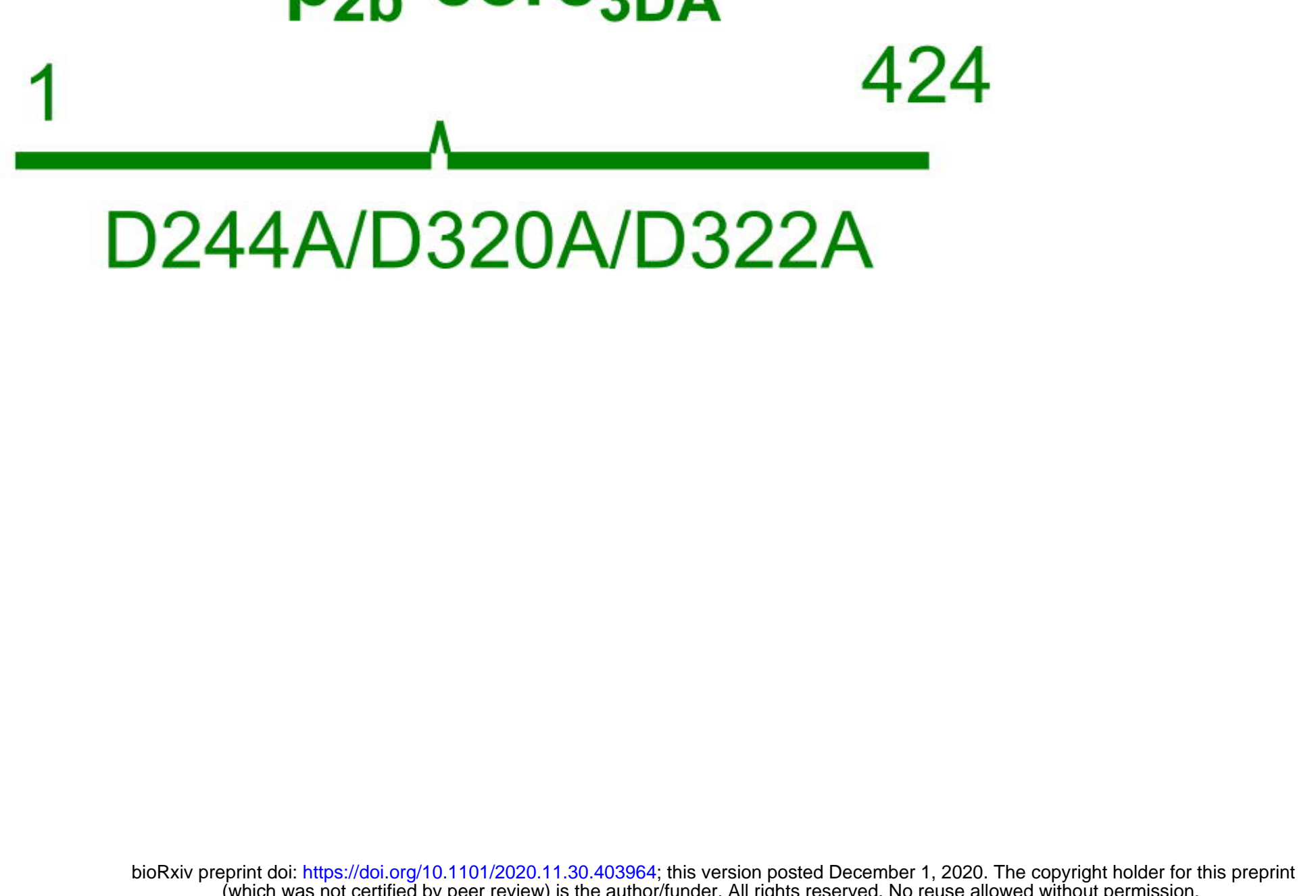

R $\quad \beta_{2 b}$-wt, no Rad

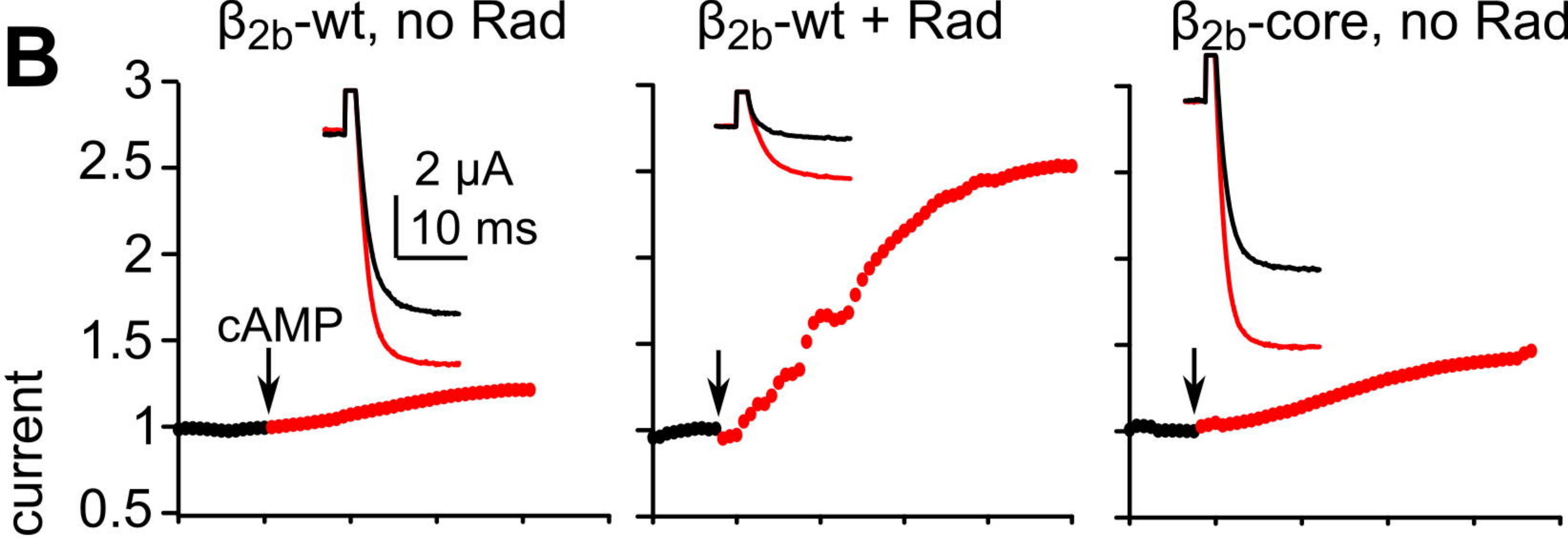

욜

$\beta_{2 b}$-core $3 D A$, no Rad $\beta_{2 b}$-core $3 D A+\operatorname{Rad}$
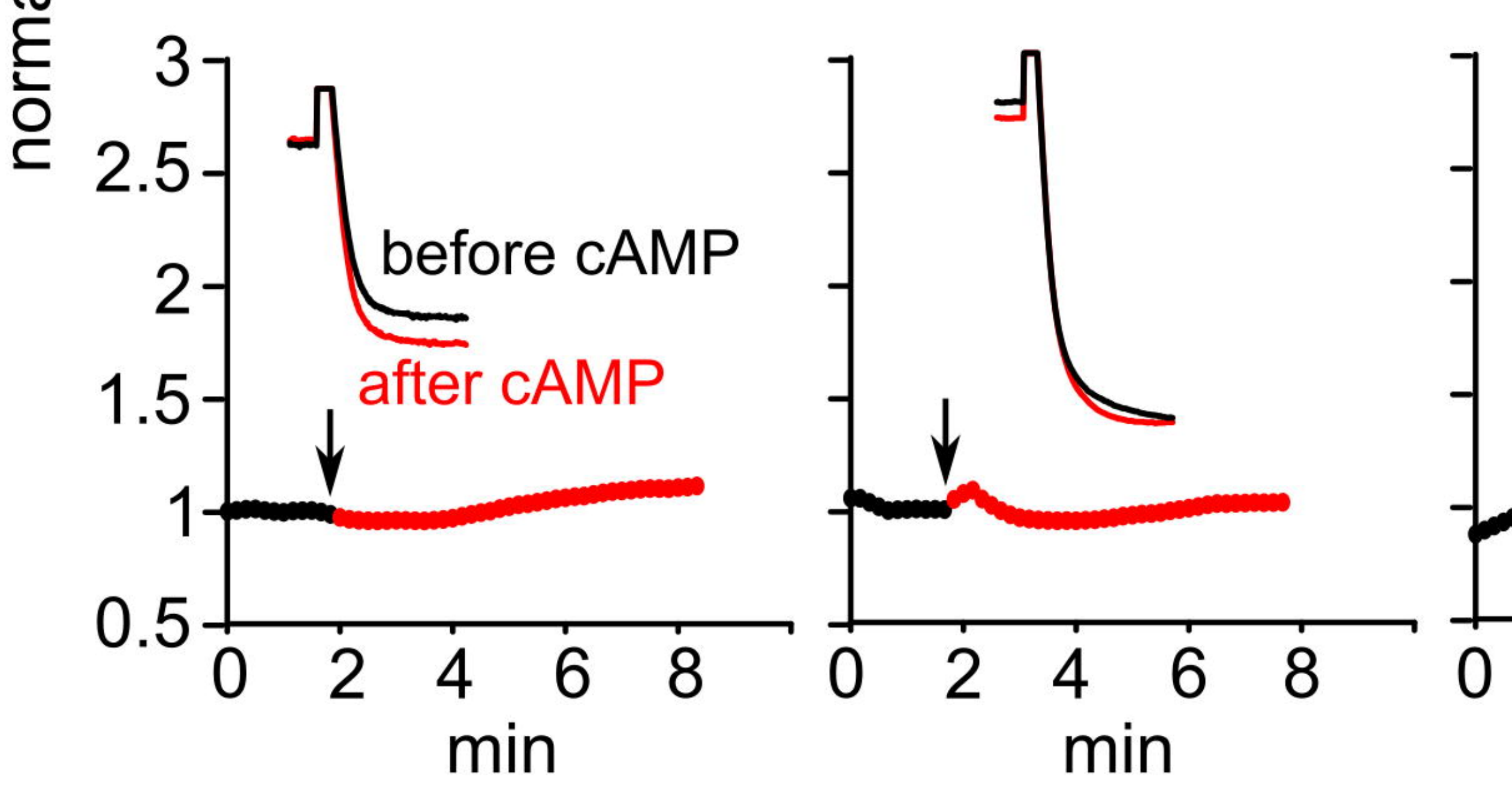

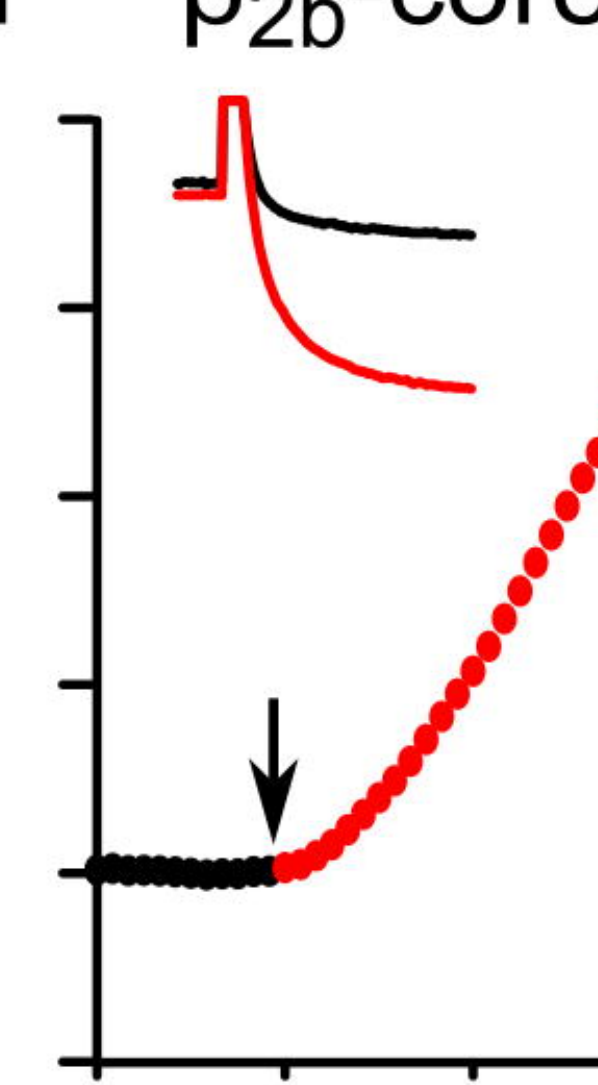

no $\beta+\operatorname{Rad}$

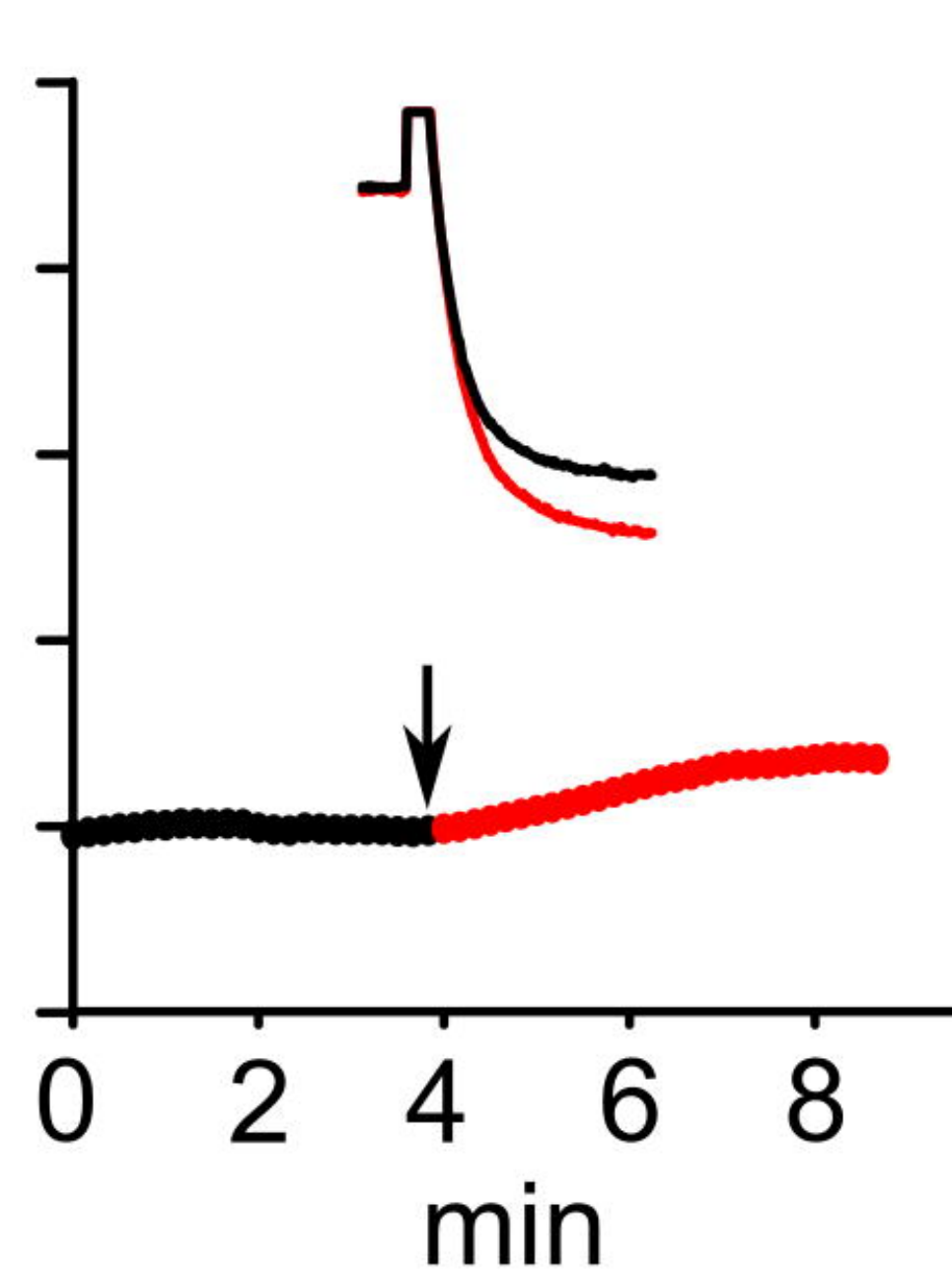

C

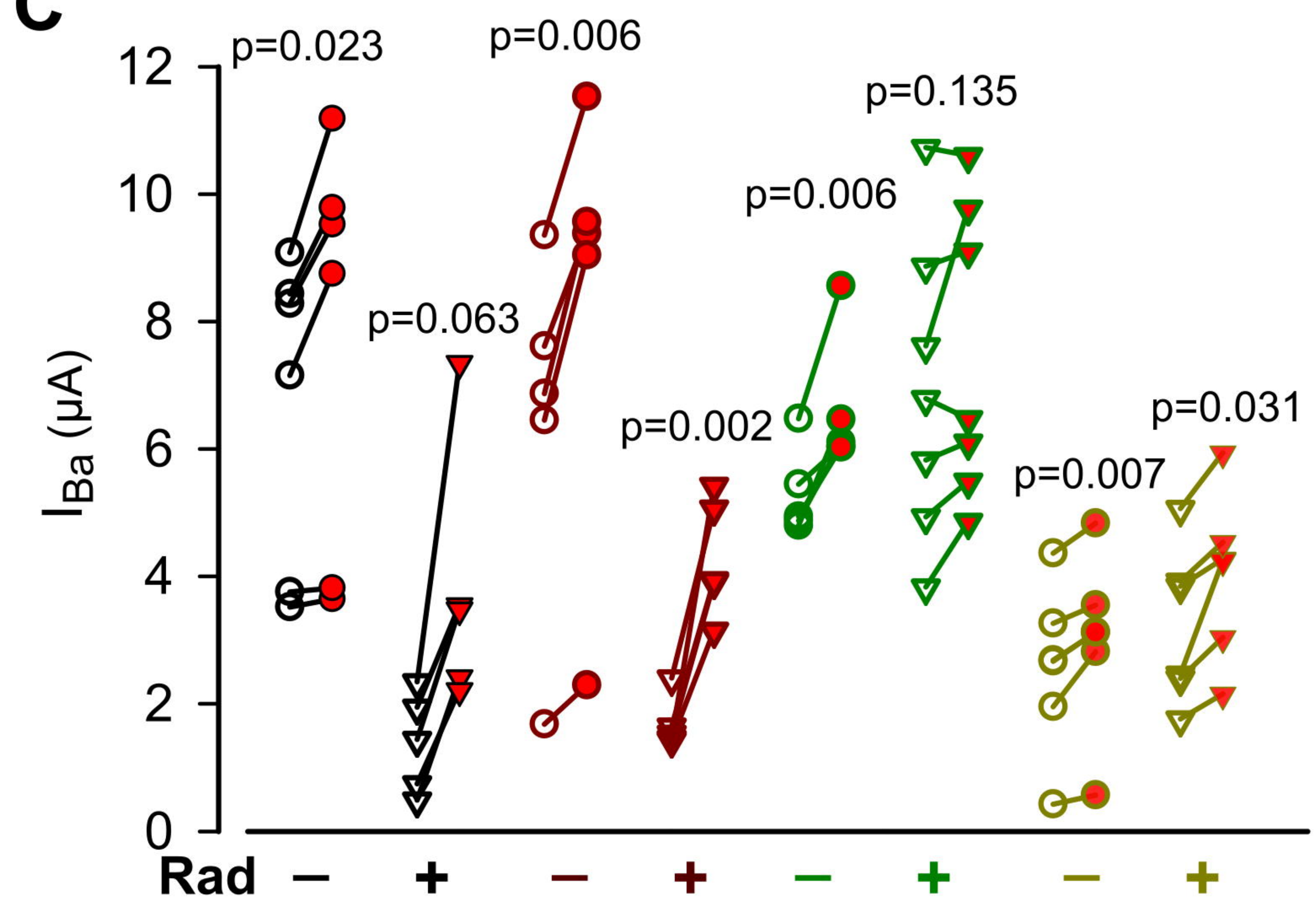

$\beta_{2 b} \quad w t$

\begin{abstract}
core
\end{abstract}
D

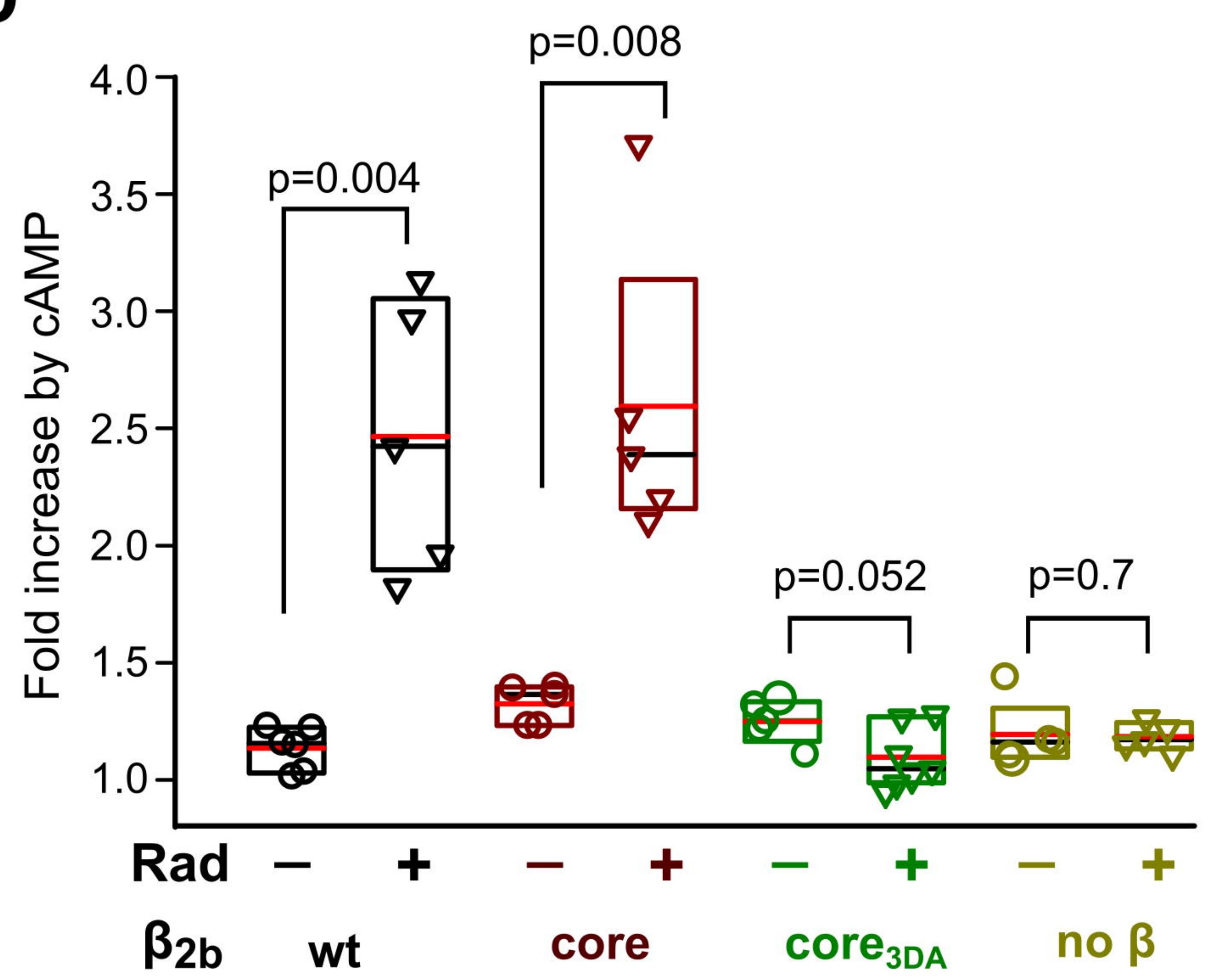




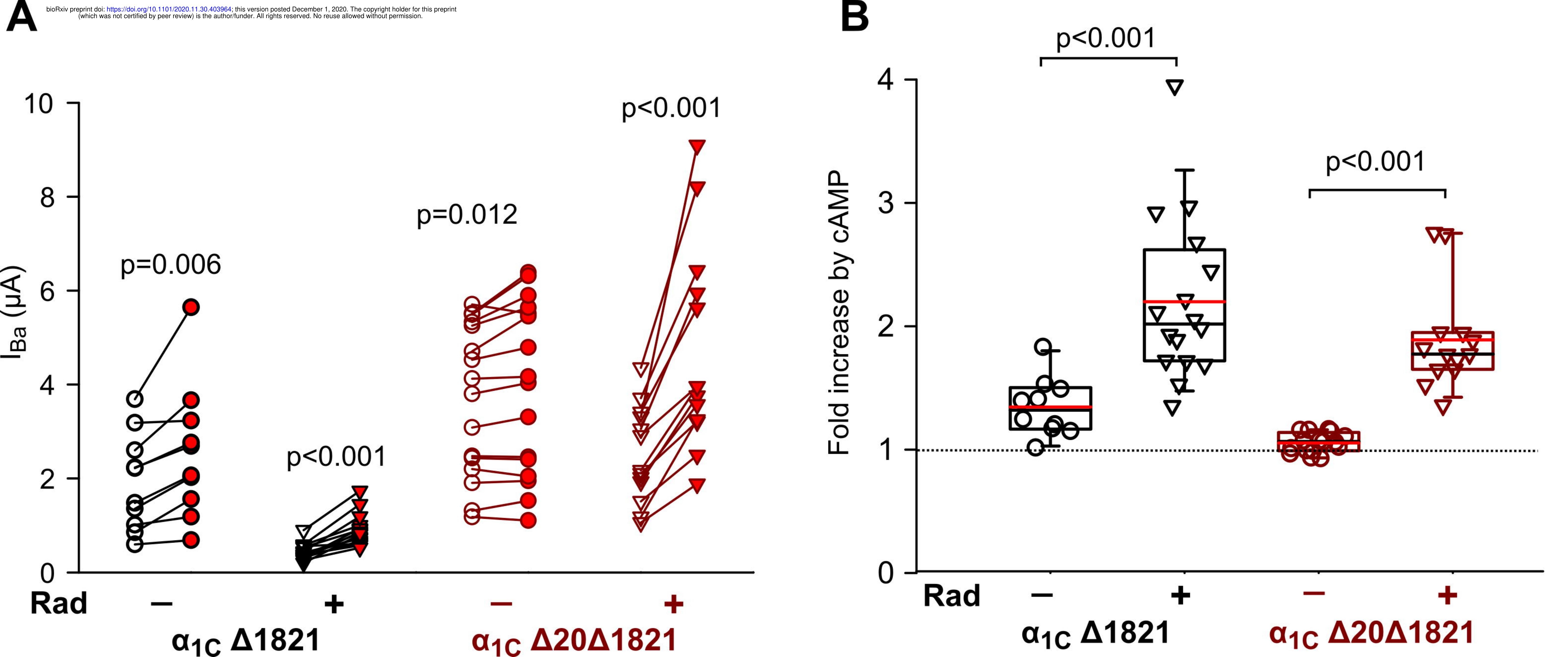



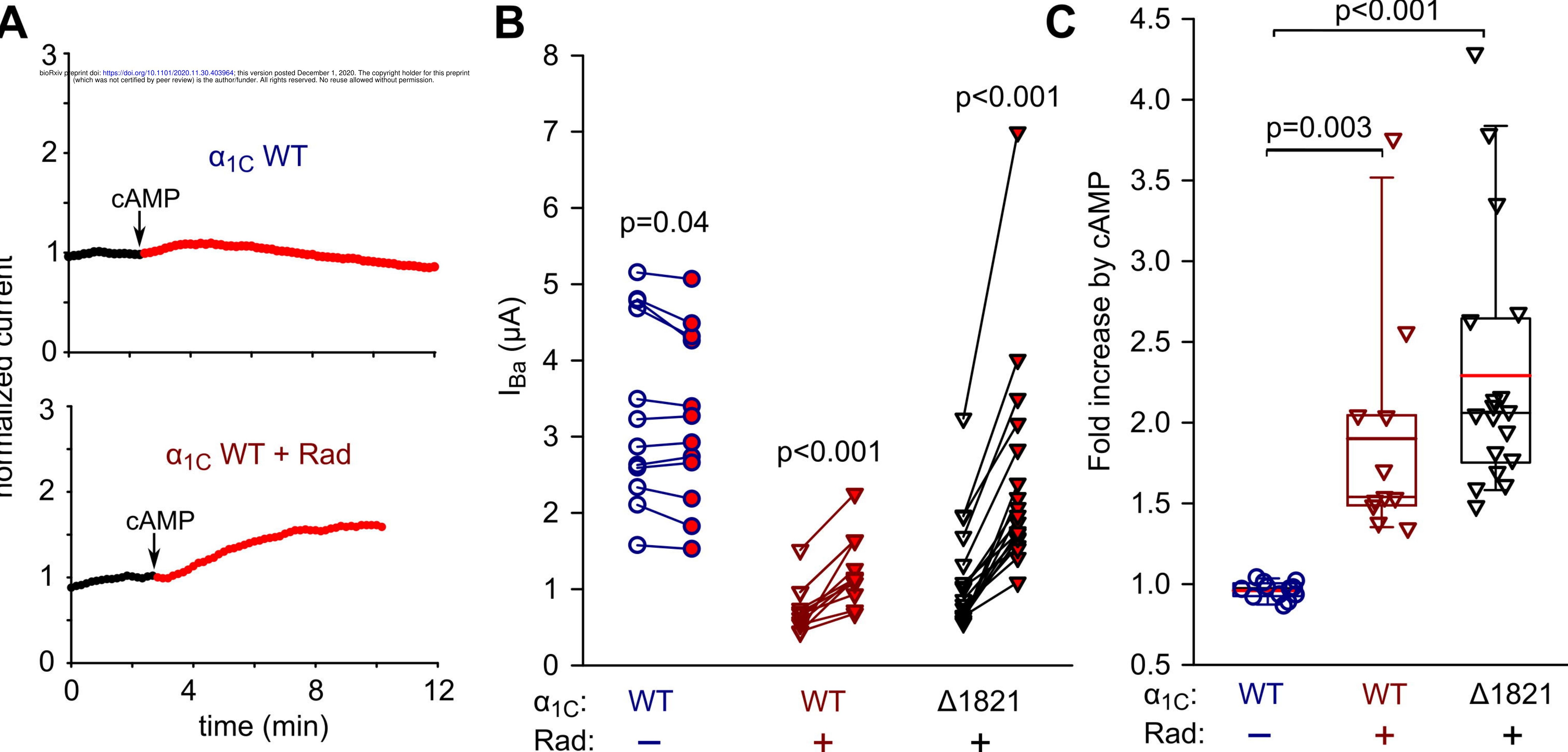
A
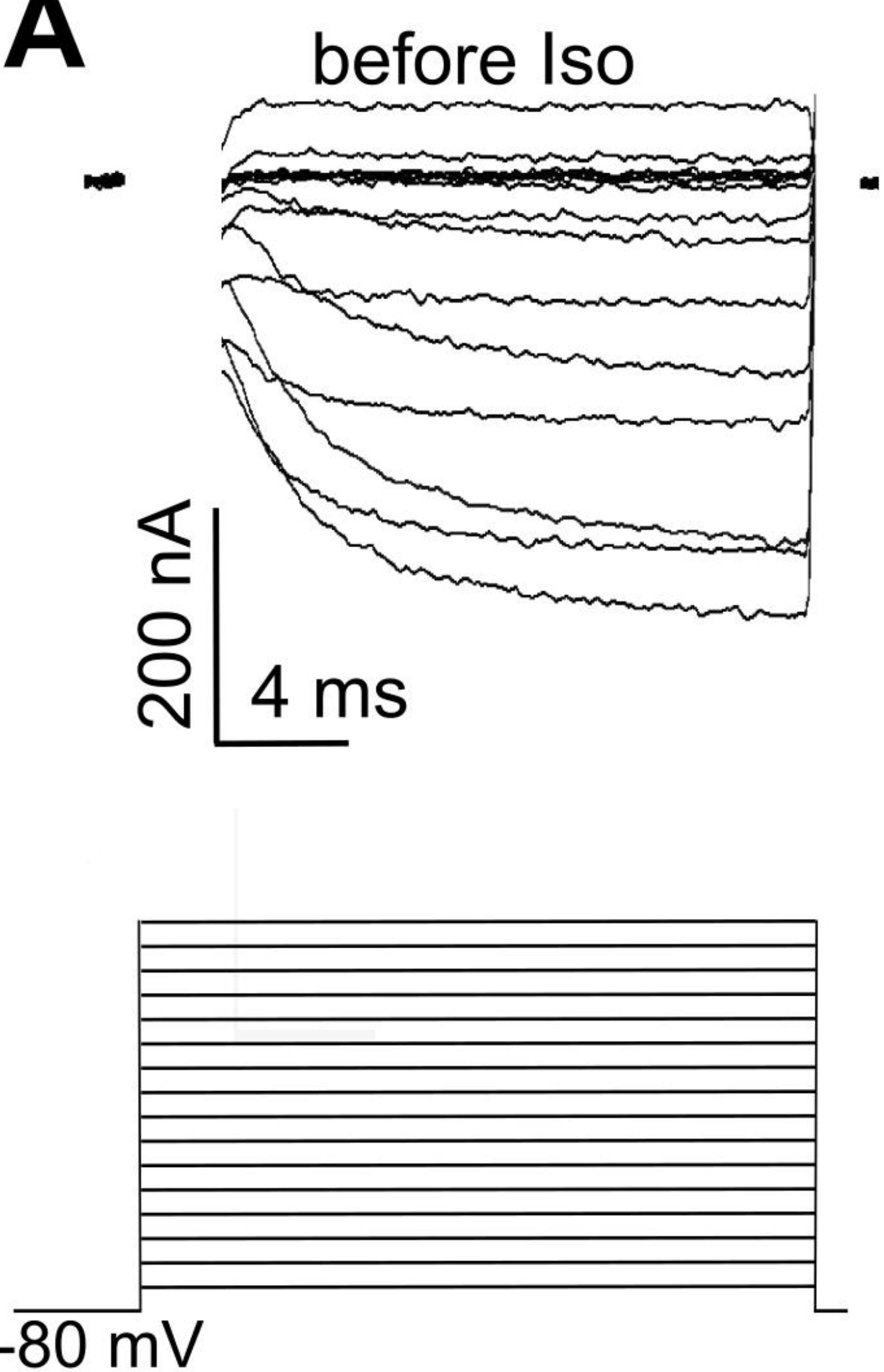

$-80 \mathrm{mV}$

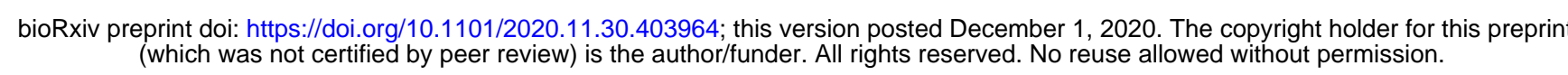

D

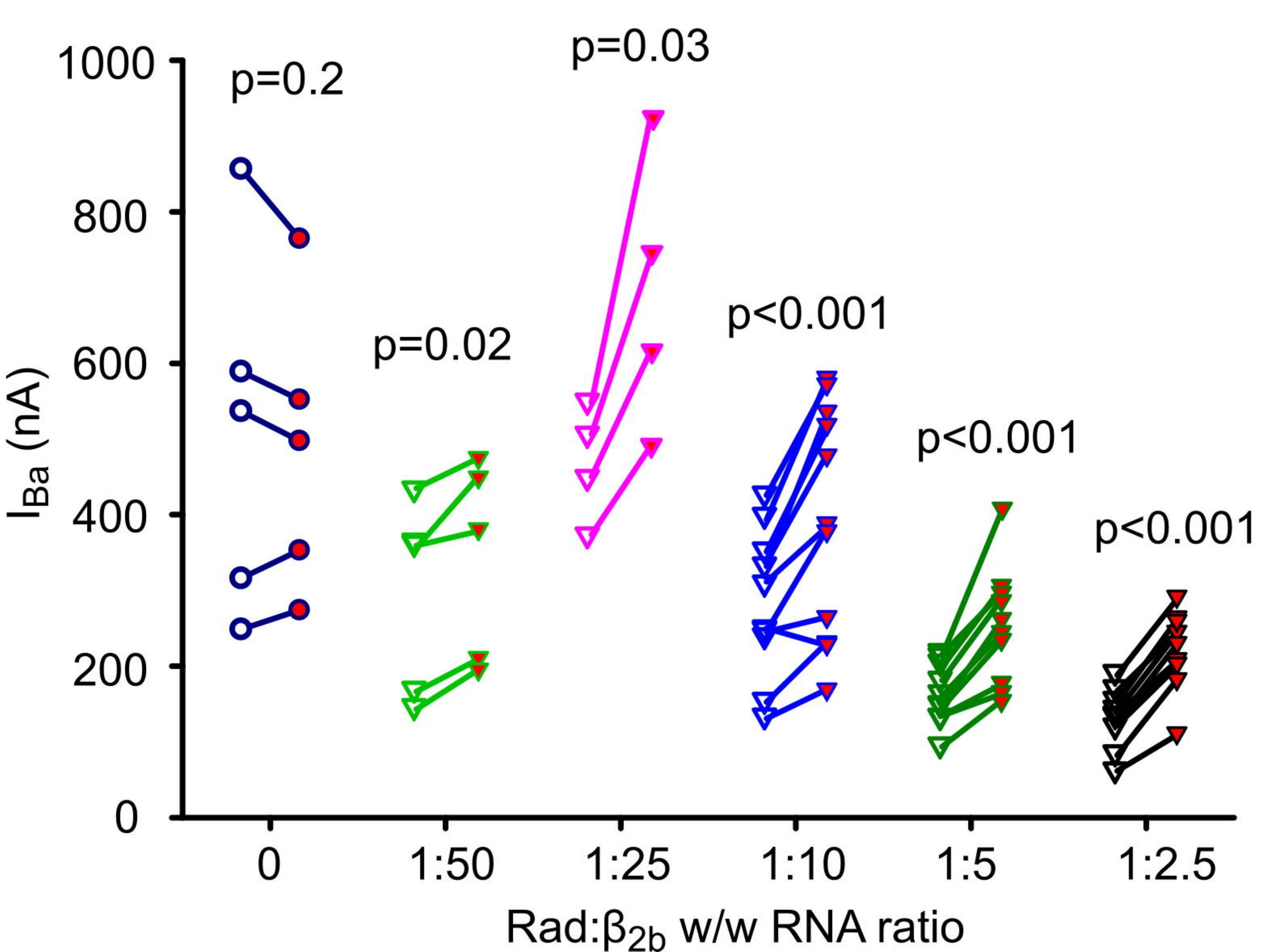

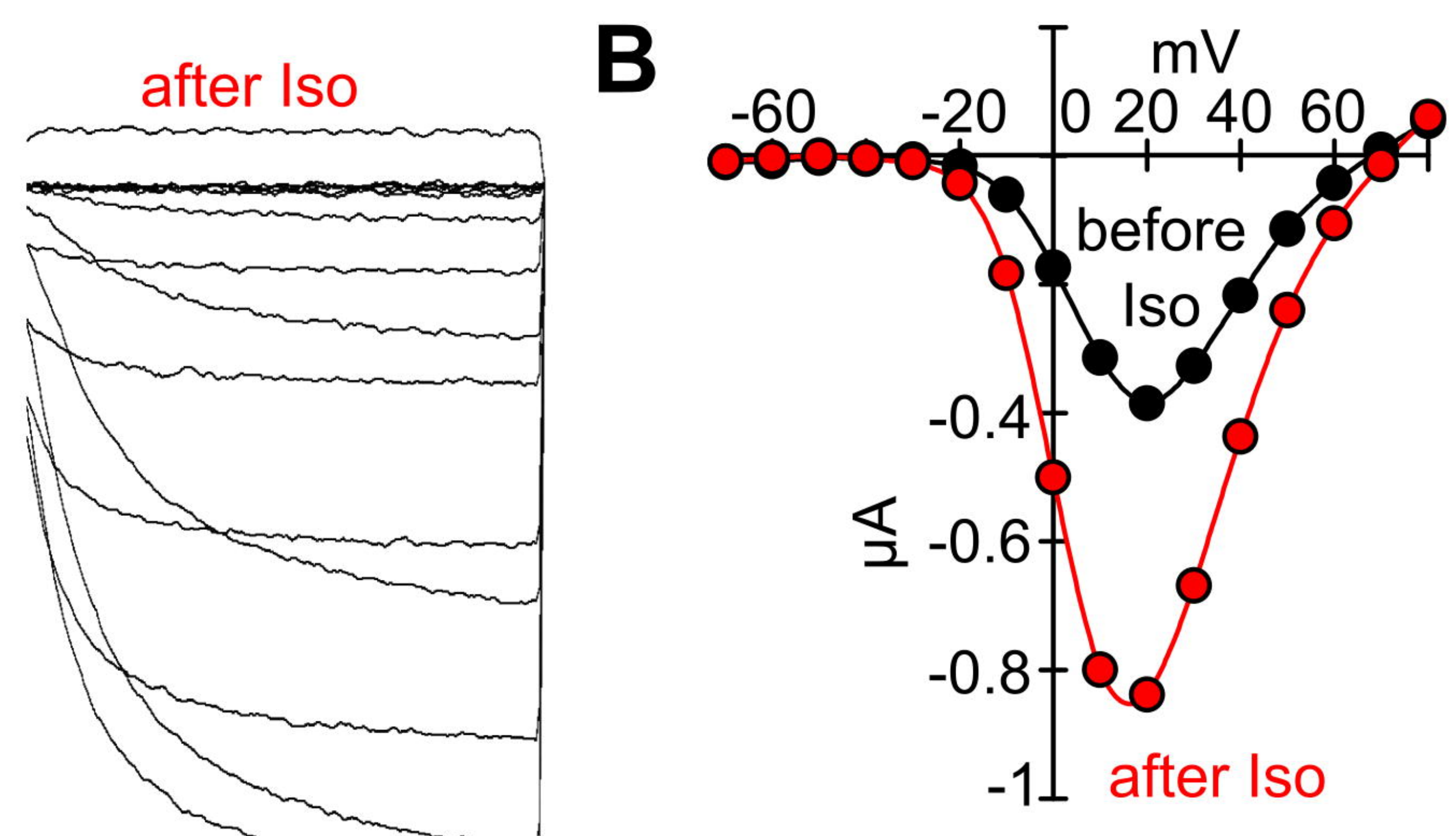

\begin{tabular}{lccc} 
& Before Iso & After Iso & $\mathbf{p}$ \\
\hline Gmax & $6.9 \pm 0.9$ & $17.8 \pm 1.5$ & $<0.001$ \\
$\mathbf{V}_{\text {rev }}$ & $61 \pm 1.4$ & $64 \pm 0.3$ & 0.075 \\
$\mathbf{V}_{\mathbf{1} / \mathbf{2}}$ & $8.7 \pm 0.7$ & $3.8 \pm 0.4$ & 0.016 \\
$\mathbf{K}_{\mathbf{a}}$ & $6.8 \pm 0.13$ & $6.6 \pm 0.14$ & 0.3
\end{tabular}

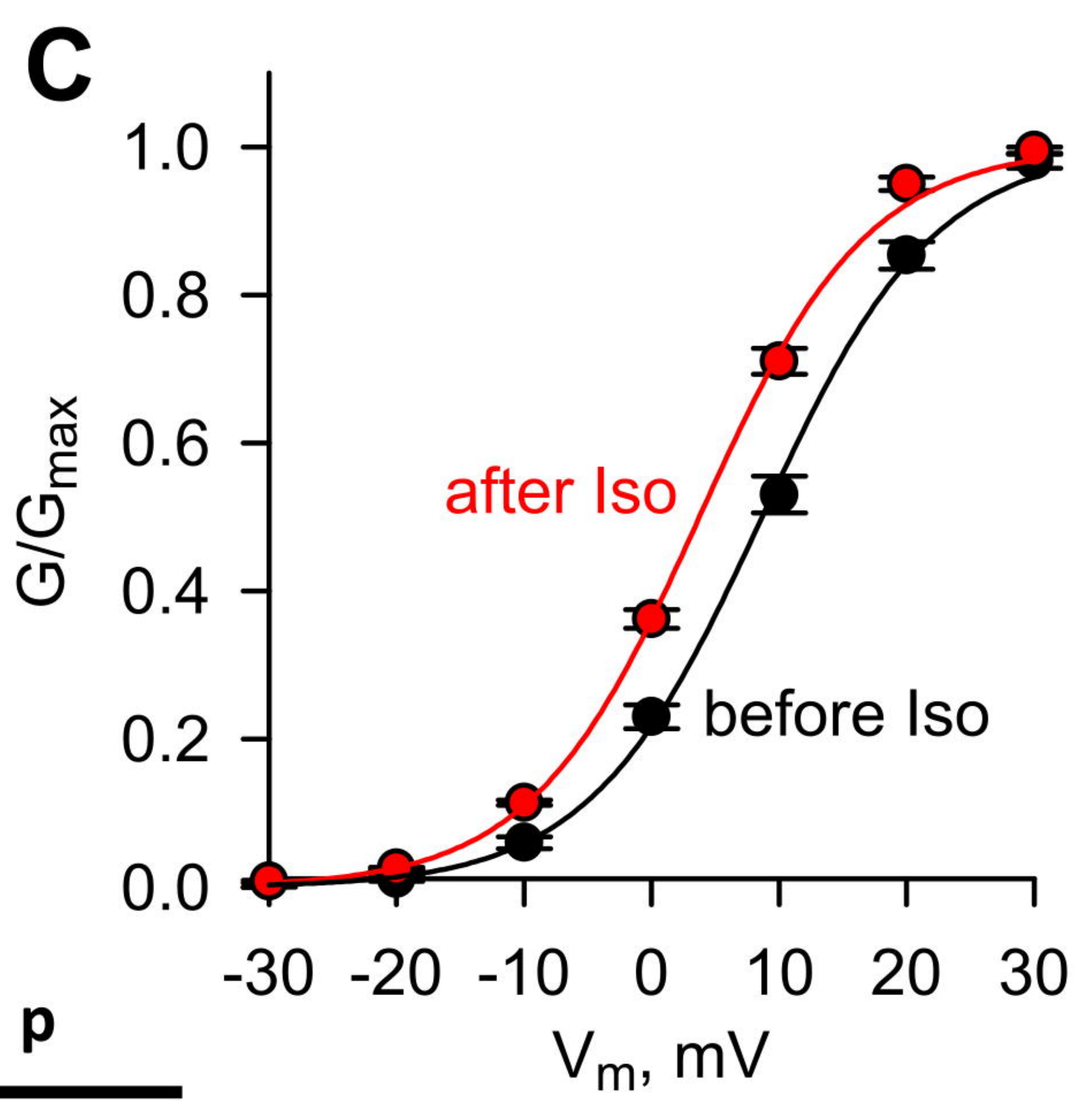

E

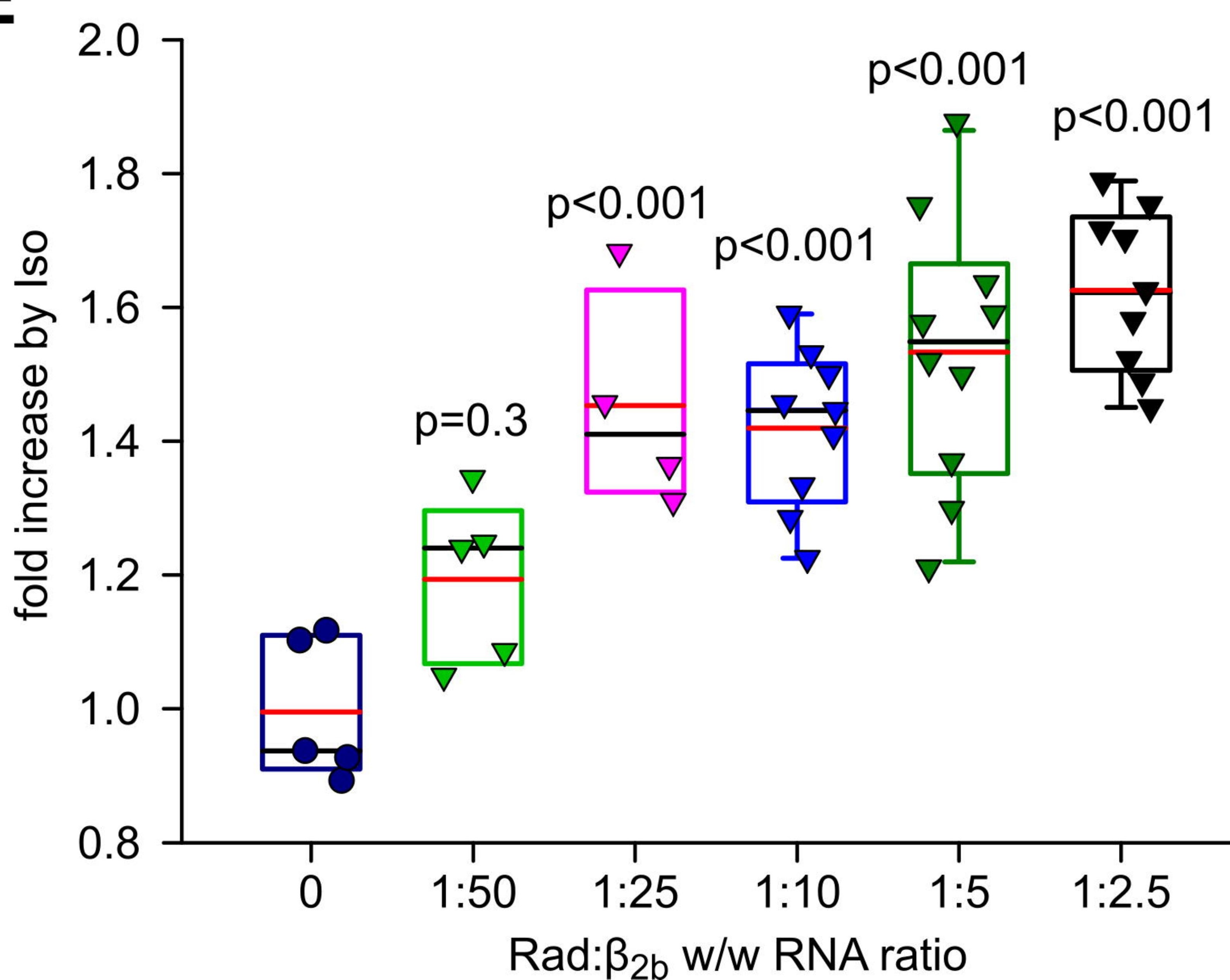


A
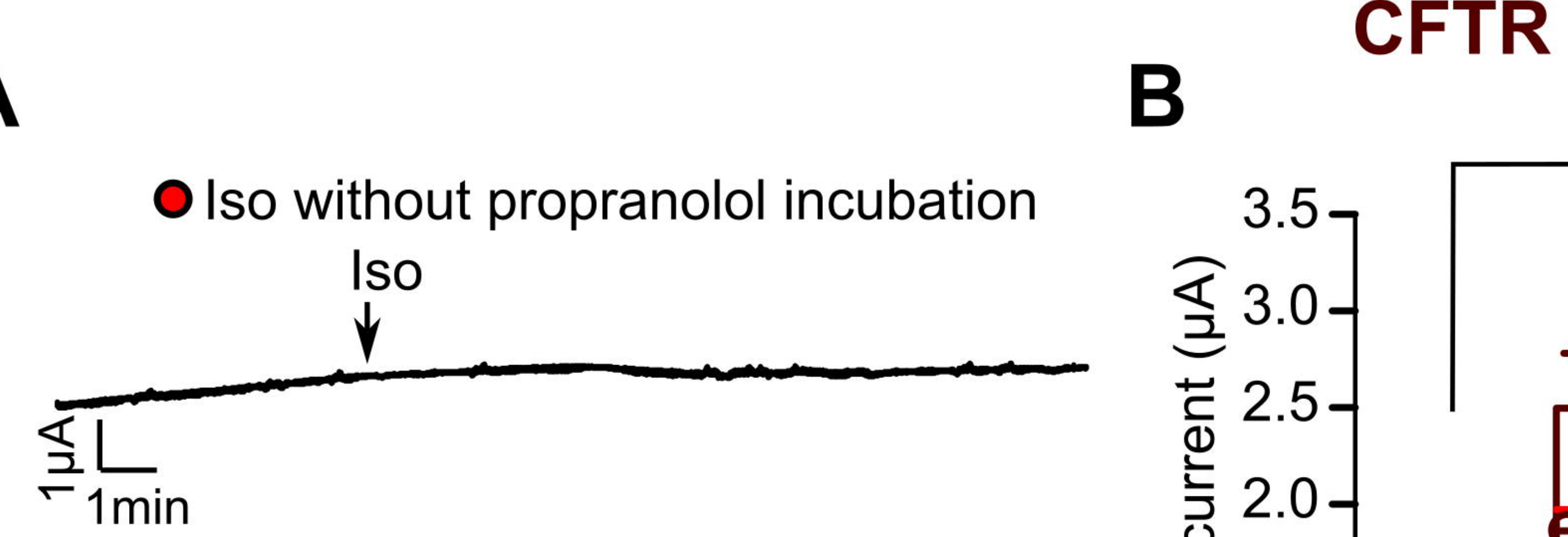

O Iso after propranolol incubation

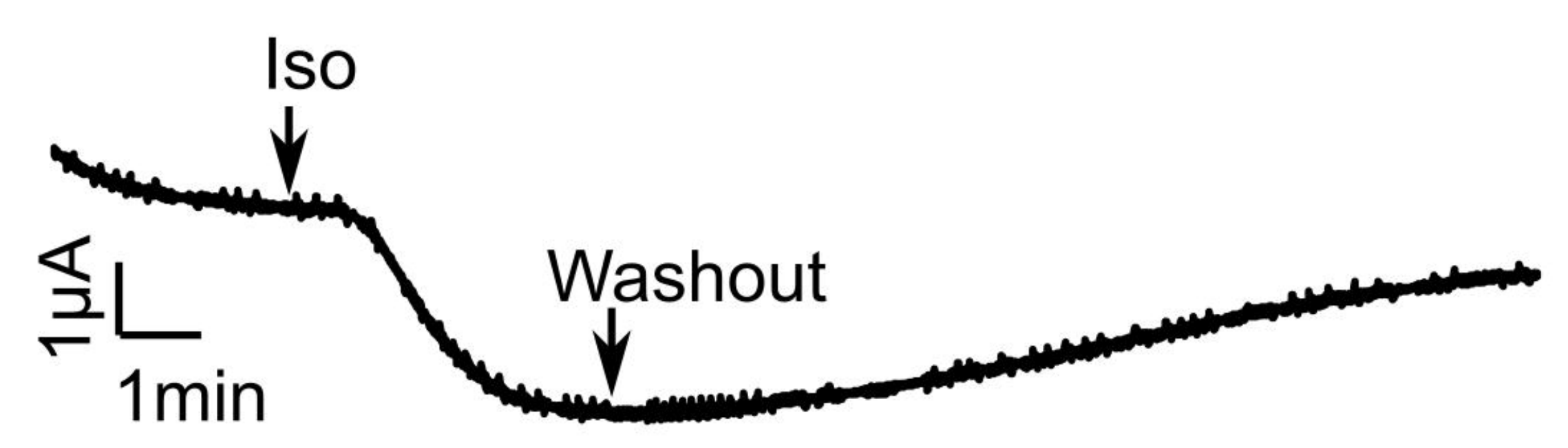

B

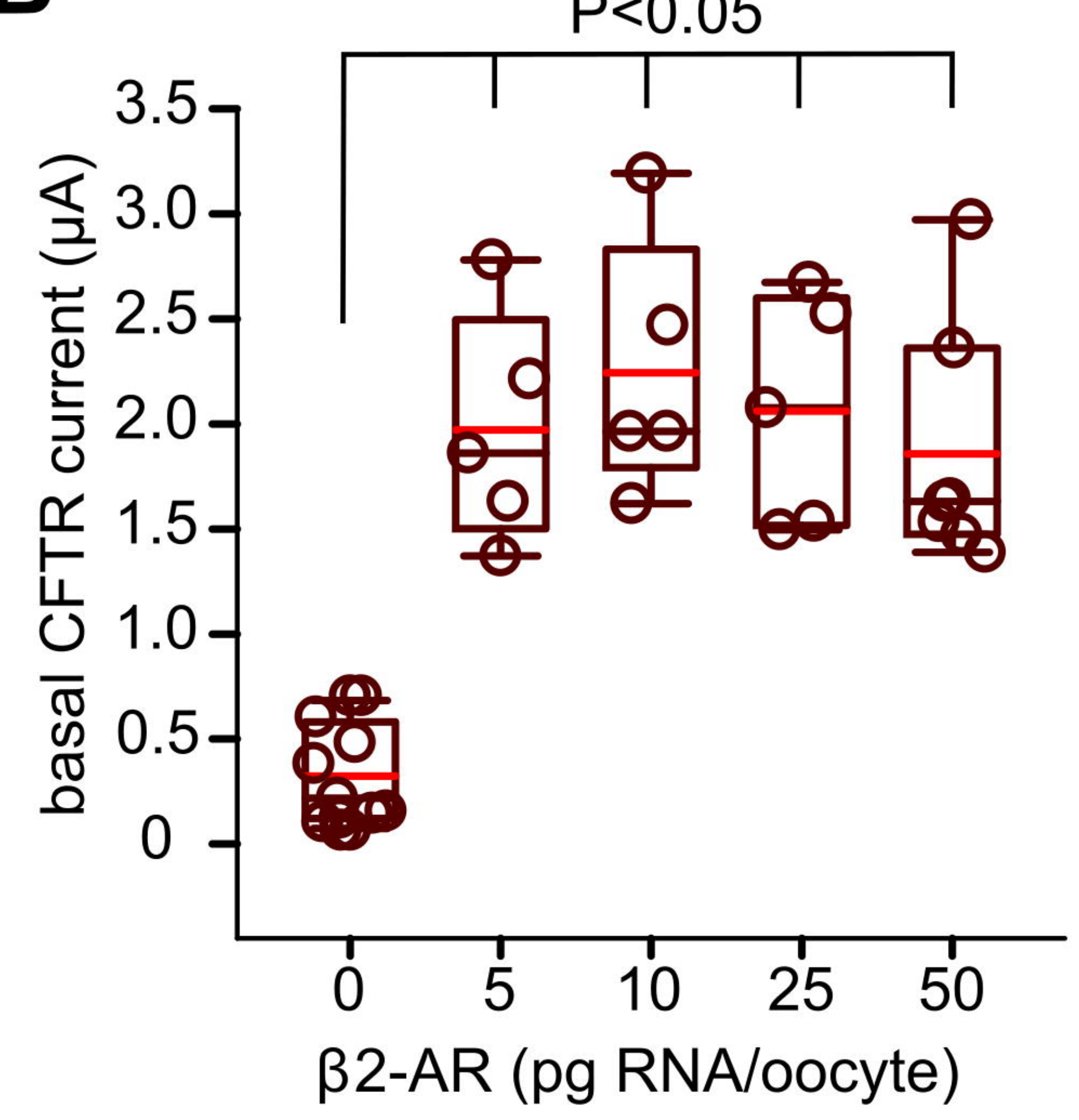

C

O Iso without propranolol incubation O Iso after propranolol incubation

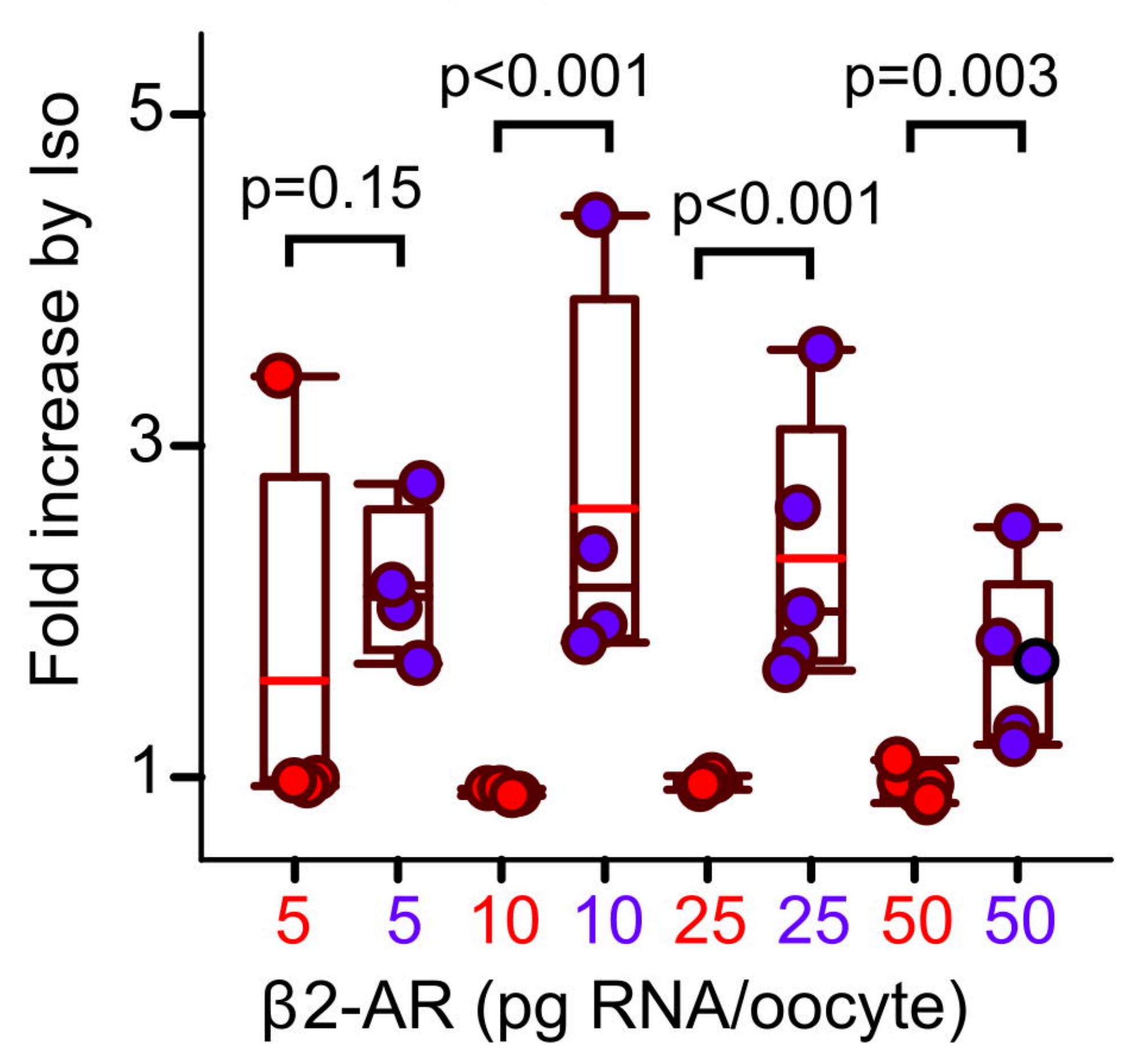

\section{$\alpha_{1 C} \Delta 1821+\operatorname{Rad}+\beta 2-A R$}

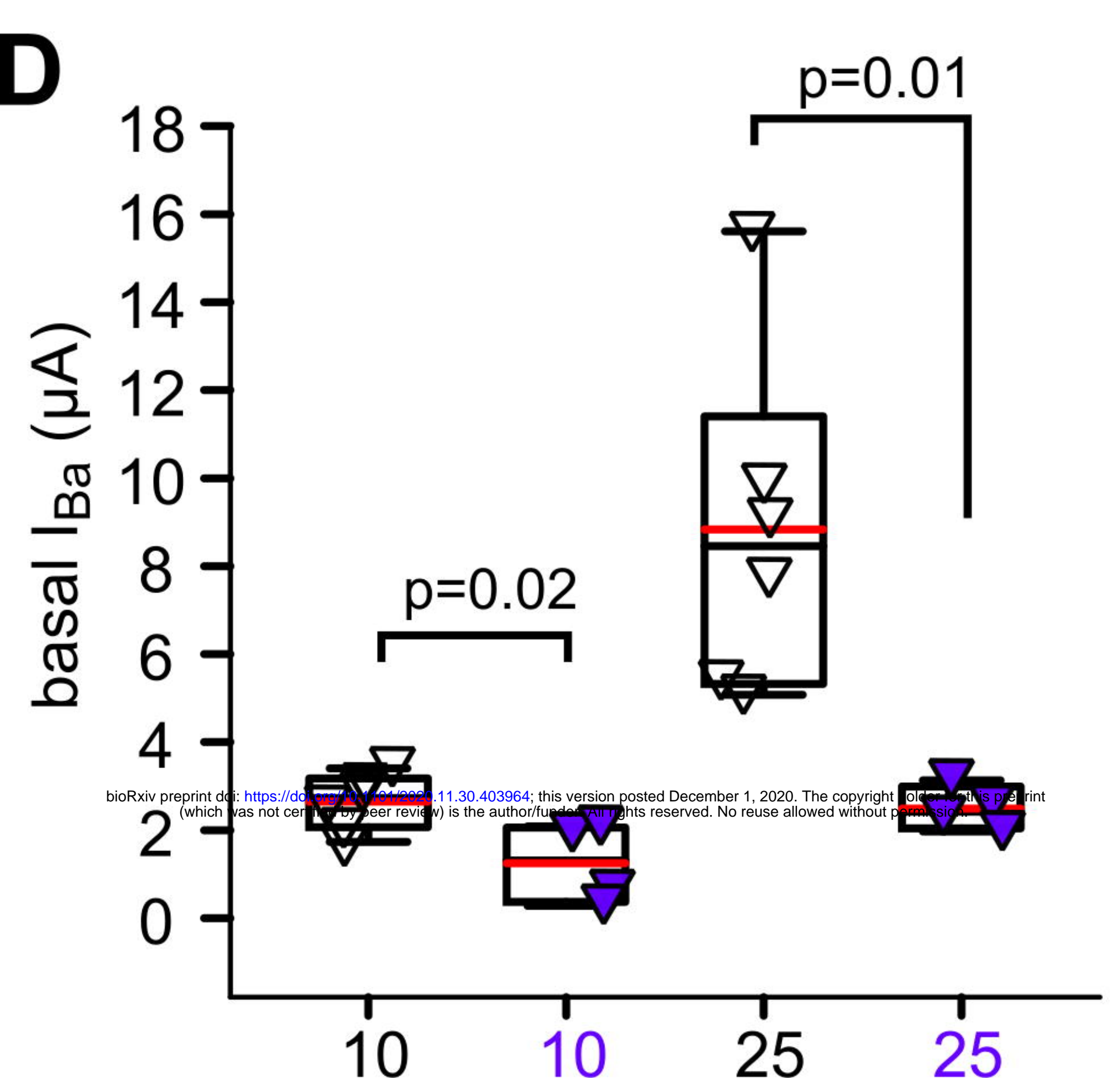

B2-AR (pg RNA/oocyte)

$\nabla$ no proporanolol incubation

$\nabla$ after proporanolol incubation
E

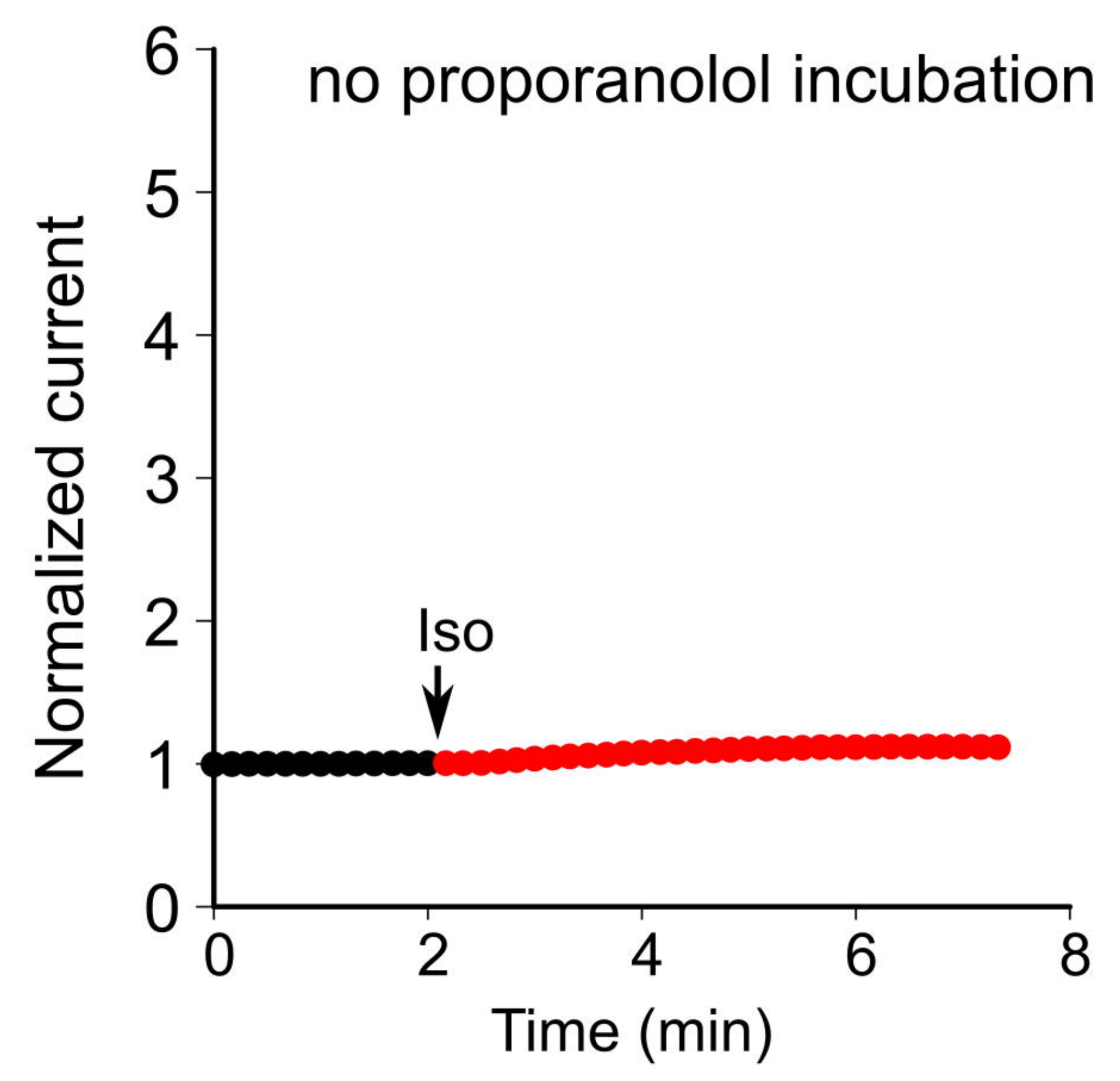

G

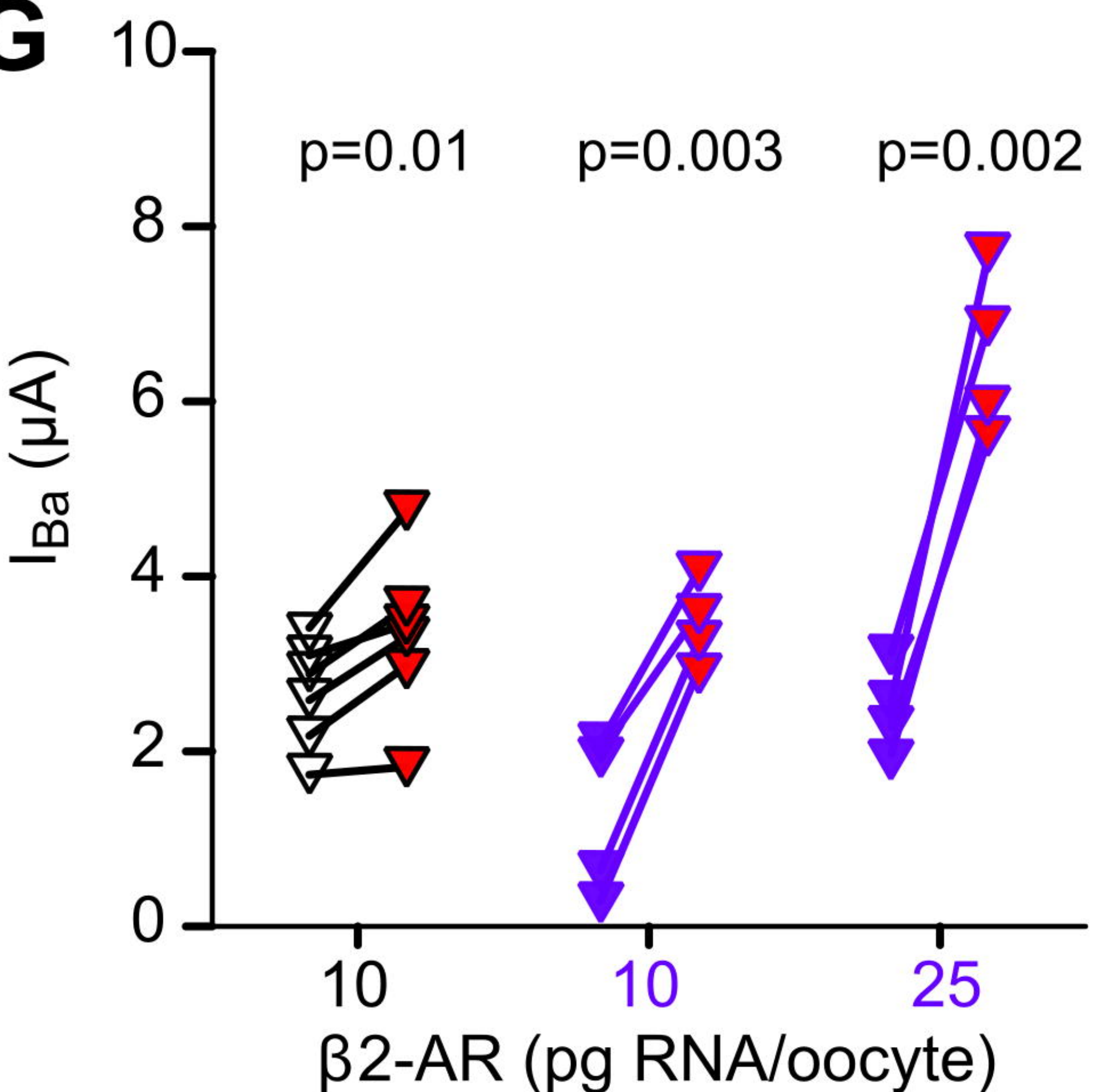

$F$
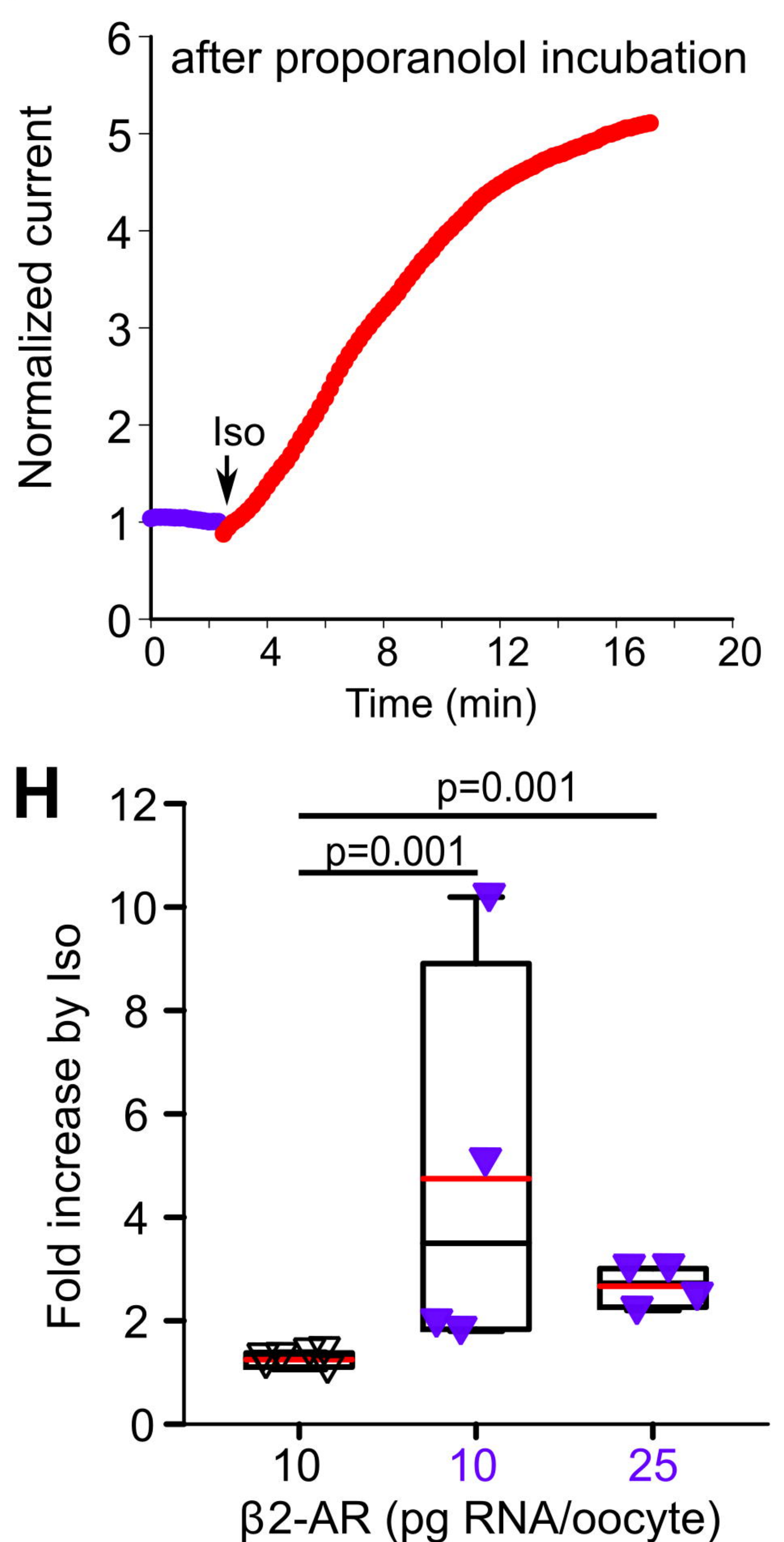